\title{
Design Considerations for Vaccine Trials with a Special Focus on COVID-19 Vaccine Development
}

\author{
Jie Chen ${ }^{* 1}$ And Naitee Ting $^{2}$ \\ ${ }^{1}$ Biostatistics and Research Decision Sciences, Merck \& Co., Inc., North Wales, Pennsylvania, USA \\ ${ }^{2}$ Biostatistics and Data Science, Boehringer-Ingelheim, Ridgefield, Connecticut, USA.
}

\begin{abstract}
The COVID-19 pandemic has triggered explosive activities in searching for cures, including vaccines against the SARS-CoV-2 infection. As of April 30, 2020, there are at least 102 COVID-19 vaccine development programs worldwide, the majority of which are in preclinical development phases, five are in phase I trial, and three are in phase I/II trial. Experts caution against rushing COVID-19 vaccine development, not only because the knowledge about SARS-CoV-2 is lacking (albeit rapidly accumulating), but also because vaccine development is a complex, lengthy process with its own rules and timelines. Clinical trials are critically important in vaccine development, usually starting from small-scale phase I trials and gradually moving to the next phases (II and III) after the primary objectives are met. This paper is intended to provide an overview on design considerations for vaccine clinical trials, with a special focus on COVID-19 vaccine development. Given the current pandemic paradigm and unique features of vaccine development, our recommendations from statistical design perspective for COVID-19 vaccine trials include: (1) novel trial design (e.g., master protocol) to expedite the simultaneous evaluation of multiple candidate vaccines or vaccine doses, (2) human challenge studies to accelerate clinical development, (3) adaptive design strategies (e.g., group sequential designs) for early termination due to futility, efficacy, and/or safety, (4) extensive modeling and simulation to characterize and establish long-term efficacy based on early-phase or short-term follow-up data, (5) safety evaluation as one of the primary focuses throughout all phases of clinical trials, (6) leveraging real-world data and evidence in vaccine trial design and analysis to establish vaccine effectiveness, and (7) global collaboration to form a joint development effort for more efficient use of resource and expertise and data sharing.
\end{abstract}

Keywords adaptive design; human challenge trials; master protocol; sample size; SARS-CoV-2; vaccine efficacy

\section{Introduction}

Coronaviruses, named for the crown-like spikes on their surface, are "a large family of viruses which may cause illness in animals or humans. In humans, several coronaviruses are known to cause respiratory infections ranging from the common cold to more severe diseases such as Middle East Respiratory Syndrome (MERS) and Severe Acute Respiratory Syndrome (SARS)" (WHO, 2020a). The most recently discovered coronavirus, a novel SARS coronavirus 2 (SARSCoV-2), which was unknown before the outbreak in Wuhan, China, in December 2019, causes coronavirus disease COVID-19 which became a global pandemic affecting millions of people in over 210 countries worldwide (WHO, 2020a) with fatality rates ranging from $0.3 \%$ to $11.4 \%$ (Oke

\footnotetext{
${ }^{*}$ Corresponding author. Email: jiechen0713@gmail.com.
} 
and Heneghan, 2020). Active researches have been carried out globally to study the epidemiology (e.g., distribution, transmission, control and prevention) and clinical management of COVID-19, to sequence and synthesize viral DNA/RNA for vaccine development, and to conduct clinical trials for anti-COVID-19 drugs and anti-SARS-CoV-2 vaccines. To date, there are no specific antiviral drugs to treat COVID-19 or vaccines to prevent SARS-CoV-2 infection. However, according to the World Health Organization (WHO), as of April 30, 2020, there are at least 102 ongoing COVID-19 candidate vaccine development programs worldwide, the majority of which are in preclinical development, five are in phase I clinical trial, and three are in phase I/II clinical trial (WHO, 2020c).

A vaccine works on the human immune system to elicit immune responses to prevent infection and the disease caused by certain pathogens such as SARS-CoV-2 and/or to reduce disease severity they may cause such as COVID-19. In order to better design a vaccine clinical trial, it is essential to understand the biological mechanisms of different types of vaccines and their main features including immunogenicity and safety. Recent scientific and technological advances have brought much insight in understanding the mechanism of microbes causing human diseases and have helped the development of various types of vaccines. According to the National Institute of Allergy and Infectious Diseases (NIAID), there are basically three types of vaccines (https://www.niaid.nih.gov/research/vaccine-types):

1. Whole-pathogen vaccines: These vaccines consist of entire pathogens or microbes such as viruses that are inactivated or weakened so that they cannot cause diseases when entering into the body. This type of vaccines includes inactivated vaccines and live-attenuated vaccines. The inactivated vaccines are produced by killing microbes using physical (e.g., heat), chemical (e.g., formaldehyde), or radiation methods to destroy the pathogen's ability to replicate, but to keep its antigen "intact" so that it can be recognized by the human immune system. The live-attenuated vaccines are obtained by passing a virus through a non-human host (e.g., chick embryos) to produce a version of the virus that cannot replicate in a human host, but that can still be recognized by the immune system. While both inactivated and live-attenuated vaccines have their unique advantages and disadvantages, the latter can elicit strong, often life-long, immune responses with just one or two doses. Examples of wholepathogen vaccine candidates against SARS-CoV-2 infection are the inactivated candidate vaccine by Beijing Institute of Biological Products/Wuhan Institute of Biological Products and the live-attenuated vaccine by Codagenix/Serum Institute of Indian, both are under preclinical evaluation (Chen et al., 2020b; WHO, 2020c). The main concerns over live-attenuated vaccines are the possibility of reverting to a non-attenuated, pathogenic phenotype in vaccinated individuals, particularly those with compromised immune system, and possible spread of the vaccine virus in the population (Robert-Guroff, 2007).

2. Component vaccines: Component vaccines consist of only a specific component (subunit) or antigen (protein or carbohydrate) from the virus, that can best stimulate the human immune system. Since antigens alone often may not be able to induce sufficient long-term immunity, component vaccines usually require adjuvants to elicit strong protective immune response. So far, there are a few SARS-CoV-2 component vaccine candidates in preclinical development that are based on the so-called S protein, a spike protein on the surface exposure that can be directly recognized by the host immune system, e.g., the recombinant protein nanoparticle vaccine candidate by Novavax and COVID-19 XWG-03 truncated S proteins vaccine candidate by Innovax/Xiamen Univ./GSK, both are in preclinical evaluation (WHO, 2020c). In general, the incidences of adverse reactions due to administration of component vaccines are relatively low because only part of the pathogen is injected into the body. However, 
component vaccines may be less immunogenic than live attenuated vaccines. In addition, antigens stimulating the immune system must be pre-identified and broad enough to cover the majority of the pathogenic virus (Vartak and Sucheck, 2016).

3. Nucleic acid vaccines: These vaccines contain an injection of a few pieces of a microbe's messenger RNA (mRNA) to encode antigens or DNA strands that can instruct the immune system to produce antigens against which an immune response is induced. The mRNA vaccines use novel technologies for genomic screening, modification, optimization, and selection of delivery system and have been reported to be highly potent and safe for administration with low manufacturing costs (Pardi et al., 2018). Although DNA vaccines are generally believed to be superior over mRNA vaccines in terms of formulation for vaccine stability and delivery efficiency, they need to enter into the nucleus, which may cause concerns of vector integration and mutation in the host genome (Liu, 2019; Zhang et al., 2020). Theoretically, nucleic acid vaccines would in general be efficient, producing a stimulation of long-term immune response. However, many of such vaccines are still under investigation and none of them have been licensed for human use. The vaccine co-developed by Moderna and NIAID against SARS-CoV-2 in phase I trial is an mRNA vaccine that encodes $\mathrm{S}$ protein (clinicaltrials.gov, NCT04283461). So far, there are several SARS-CoV-2 DNA vaccines under preclinical development (WHO, 2020c).

For a virus vaccine to be used in humans, it has to be safe, immunogenic, stable, and capable of inducing sufficient immunity. To meet these requirements, a vaccine development program must provide a comprehensive understanding of (1) virus molecular and structural biology and pathogenesis, (2) the complexity and plasticity of major viral antigens, (3) the protective role of antiviral $\mathrm{B}$ and $\mathrm{T}$ cell mediated immune responses, and (4) any potential side effects that may be associated with excessive immune response, adjuvants, manufacturing processes, and administration routes (Enjuanes et al., 2016); see also Section 3 for more discussion on immune responses and immunogenicity of exogenous pathogens.

This paper provides an overview on design considerations for vaccine clinical trials, with a special focus on COVID-19 vaccine development. Section 2 presents general considerations for vaccine studies and discusses the rationale on why vaccine development cannot be rushed. Section 3 briefly introduces human immune responses upon exposure to exogenous pathogens, immunogenicity measures of a vaccine, and considerations in phase I and phase II vaccine trials. More discussions on phase III trials, including endpoints, study population, randomization, adaptive design options, and sample size determination are given in Section 4. Section 5 presents rationale, design considerations and challenges in using real-world data and evidence in vaccine effectiveness trials. Considerations for vaccine safety evaluation including a general strategy and designing a vaccine trial with a major safety endpoint as a primary hypothesis are presented in Section 6. Other considerations, such as assessment of correlates of protection, natural herd immunity and vaccine direct and indirect effects, and waning efficacy, are discussed in Section 7. Finally, some concluding remarks and recommendations are given in Section 8.

\section{General Considerations in Vaccine Development}

Vaccines differ from drugs (including therapeutic biologics) in a variety of ways, such as safety standard, target population, development process, regulatory requirement, and post-approval life-cycle management. Full understanding of these differences is crucial in designing vaccine trials. In general, the following special considerations must be taken in developing a vaccine 
product:

- High safety standard: Unlike drugs or therapeutic biologics, prophylactic vaccines are usually given to healthy populations, some of whom may be vulnerable children, older adults, and/or special populations (e.g., service men and women). Therefore, safety standard is generally higher for vaccines than for drugs. Some vaccines are universally recommended and as a result are administered to a large number of people. Hence, "first do no harm" is the widespread acceptable principle in public health and a much lower risk tolerance is expected.

- Immune responses as surrogate markers: Vaccines work through stimulating the human immune system to first produce immune responses (humoral immunity and cellular immunity) and to fight against invading pathogens (e.g., viruses) and infectious diseases. The immune responses, serving as a surrogate marker, are usually measured by biological assays for the amount, classes or subclasses, and functionality of specific antibodies, in order to determine the degree, duration and scope of responses. In addition, the kinetics of immune response, e.g., seroconversion rate (transition from seronegative to seropositive) and induction of immune memory, should also be considered.

- Vaccine efficacy. Efficacy for vaccines is defined in terms of risk reduction, which can only be observed after proof of concept studies (phase IIa/IIb trials). Development decisions (go/no go, dose level, vaccine scheduling) are generally based on surrogate markers, also known as correlates of protection, which are often unknown until after phase III trials, especially in COVID-19 vaccines. Therefore, the use of quantitative methods to establish correlates of protection in terms of vaccine efficacy is important for early decisions.

- Varying observational time: The impact of active vaccination on the immune system is virtually life-long through memory B and T cell generation. However, some biologically plausible adverse reactions may occur only in a short period of time after vaccination, e.g., intussusception associated with rotavirus vaccine mostly occurs 7-42 days post-vaccination (Chen et al., 2010), and some others occur within years post vaccination, e.g., Dengvaxia ${ }^{\circledR}$ increasing risk of severe dengue (an unintended effect of Denvaxia ${ }^{\circledR}$ on the immune system) starting a few years after vaccination for dengue naive subjects (Thomas and Yoon, 2019). Therefore, the observational time to establish the safety profile could vary substantially for different vaccines.

- Step-down and -up development strategies: Most vaccines are developed for a specific population such as infants, elderly, or adults. In general, early safety evaluation starts with adult healthy volunteers and then steps down to adolescents and pediatric populations and steps up to geriatric populations if there are no major safety concerns. This step-down and step-up strategy may be more appropriate for COVID-19 vaccine development as these vaccines will be administered to different age groups.

- High variations in immunogenicity: Vaccines are biologically derived and variations in biological activities can occur, which can be further complicated by biological manufacturing process such as formulation, fermentation, and virus sensitivity to storage condition. Furthermore, immunological assays also tend to be much more variable than chemical assays.

- Vaccine adjuvants: Adjuvants are commonly used in component vaccines to produce an immune response strong enough to protect people. However, adjuvanted vaccines can cause more local reactions (e.g., redness, swelling, and pain at the injection site) and more systemic vaccine-related adverse reactions (e.g., fever, chills, and body aches) than non-adjuvanted vaccines (Marciani, 2003; WHO, 2017).

- Logistic challenges: A vaccine product contains biological ingredients which usually require 
special conditions (e.g., temperature) during manufacturing, transportation and on-site storage. These logistic challenges often not only result in high costs for manufacturers, but also hamper smaller biotech companies from engaging in vaccine research, development and manufacturing. Another consideration is that the number of people receiving a vaccine is typically orders of magnitude larger than the number of people receiving any single prescription drug, which usually requires substantial capacity for rapidly scale-up manufacturing in a robust and reliable manner. This could be especially important for rolling a COVID-19 vaccine out as quickly as possible during this pandemic.

Given the above considerations, vaccine development is generally complex, lengthy, and costly, and has its own development pathway (Plotkin, 2015; Cunningham et al., 2016; Plotkin et al., 2017). Some experts urge not to rush COVID-19 vaccine development (Callaway, 2020a; Jiang, 2020; Peeples, 2020), not only because the knowledge about SARS-CoV-2 virus is lacking (albeit rapidly accumulating), such as the virus epidemiology, genomic structure, mechanism of pathogenesis, pathological immune response, but also because scientists need time to fully understand how the immune system responds to SARS-CoV-2, how long the immune response can last, and most importantly, how safe the vaccine will be, when it is widely deployed to the public. Testing vaccines on an accelerated schedule without taking the time to fully understand safety risks could bring unwarranted setbacks during the current pandemic and into the future (Jiang, 2020) and public health urgency does not override the need of scientific rigor which demands reliable results (Ellenberg et al., 2018). However, under the current pandemic paradigm, some careful design considerations, such as, master protocol, human challenge trials, appropriate choice of endpoints, study population, and adaptive design strategies (e.g., group sequential design, seamless design), could potentially help shorten the overall development time without compromising the high standard of vaccine development, which is the purpose of this paper. In addition, the key statistical considerations discussed below can also be applied to other vaccine development programs in general.

\section{Immune Response, Immunogenicity, and Early-Phase Clinical Development}

\subsection{Immune Response}

The immune system can be divided into two subsystems-the innate immune system and the adaptive immune system. While the former provides immediate, short-term, and non-specific first-line response to invading pathogens by inhibiting or controlling their replication and spread during the early phase of infection, the latter can offer rapid, more specific response tailored to a particular molecular structure of pathogens by activating B-lymphocytes (or B cells) and T-lymphocytes (or T cells). The B cells produce antigen-specific antibodies that attack or inactivate invading pathogens carrying the antigens to prevent their replication, which is often called antibody-mediated immunity, and the T cells can help the B cells secrete antibodies and attack or destroy infected body cells, which is often called cellular immunity. The activation of $\mathrm{B}$ cells with help of $\mathrm{T}$ cells can result in a much better, more effective immune response with long lasting memory (immunological memory) to prepare future challenges by the same or structurally similar pathogens, which is the mechanism of immunization (Kindt et al., 2007; Clem, 2011)

Immunization can be obtained either passively or actively, each of which can be from natural sources or artificial sources. Natural passive immunization can be achieved when an infant 
obtains maternal antibodies through placenta (maternal immunity) and artificial passive immunization is gained via the administration of pooled human immune gamma globulin and antivenin (antibodies), both of which provide temporary immunity. On the other hand, natural active immunization occurs when an unimmunized individual is exposed to a pathogenic agent that triggers the body to produce antibodies to develop long-term immunity (often called herd immunity) and artificial active immunization can be acquired through administration of vaccines, both of which produce long-term immunity due to the stimulation of the human immune system against a particular pathogenic agent (Clem, 2011).

When exogenous agent (e.g., virus) enters into the body, the immune system recognizes epitopes, small subregions on the antigen that stimulate the immune system to produce antibodies in response to the challenge. Because the antibodies are antigen-specific, any antigenic variation that results from different virus strains (a genetic variant or subtype of a virus) and/or serotypes (a variant of the same species with antigenic variation that cannot be detected by antibodies targeting other members of the same species), gene mutation, or gene recombinant may induce immunologically distinct antibodies. The recent phylogenetic analysis of 103 strains of SARS-CoV-2 identified two different types of SARS-CoV-2, namely, type L (accounting for 70 percent of the strains) and type $\mathrm{S}$ (accounting for 30 percent) (Tang et al., 2020; McIntosh, 2020). Therefore, antigenic variations must be taken into account when developing a vaccine in order to have a broader coverage of or protection against different types of strains and serotypes of SARS-CoV-2.

\section{$3.2 \quad$ Immunogenicity}

Immunogenicity can be defined as the ability of a molecule or foreign substance, such as an antigen (vaccine component), to provoke a humoral and/or cell-mediated immune response or the strength and/or magnitude of the immune response in a vaccinated individual (LerouxRoels et al., 2011; Mahanty et al., 2015), and has played a critical role in assessing the efficacy of a vaccine, especially in early clinical development when only a small number of subjects is enrolled. Immunogenicity is commonly evaluated by measuring vaccine-specific cell-mediated immunity and humoral responses elicited by vaccination. Until recently, vaccine immunogenicity studies mostly focus on the humoral immune response, i.e., on serum antigen-specific antibody level which is often believed to correlate with the degree of protection provided by the vaccine (Plotkin, 2010).

Vaccine immunogenicity is measured by immunogenicity assays, a group of biomarker assays, or simply bioassays, e.g., enzyme-linked immunospot (ELISPOT) assay and the enzyme-linked immunosorbent assay (ELISA), to quantify a potential surrogate endpoint or a correlate of protection for a candidate vaccine. Another important group of assays is functional assays, e.g., serum neutralization assay, which are serological tests used to detect the presence and magnitude of functional systemic antibodies that prevent infectivity of a virus. The serum neutralization assay is a highly predictive, sensitive, and specific test, and may be used to evaluate the level of serological cross-reactivity between virus strains or vaccine antisera and heterologous viruses that may correlate with cross-protection in the host (Gauger and Vincent, 2020).

The bioassay used for measuring vaccine immunogenicity must be biologically validated and statistically qualified with clearly defined optimal performance or operating characteristics, including: (1) accuracy (unbiasedness), (2) precision (repeatability, intermediate precision, and reproducibility), (3) ruggedness (robustness of the assay under different physical or biological conditions), (4) limit of detection (the lowest concentration that can be biologically and statis- 
tically detected), (5) quantifiable range (an interval of concentration within which the biological material, e.g., antibody, can be reliably quantified with pre-defined accuracy and precision), (6) linearity of concentration (the linear segment of the concentration-response curve over a range of concentrations), (7) specificity (an ability of an assay to quantify a particular analyte); see ICH (2005) and FDA (2015) for more descriptions on the regulatory requirements of analytic procedures and methods validation. For the design and analysis of bioassay experiments, Yang et al. (2015) provide a comprehensive review and statistical methods for assessing immunogenicity assays; see also Heyse and Chan (2016) who give a review of statistical innovations in bioassays supporting vaccine clinical development and Nauta (2020, chapters 3-4) who presents statistical methods for the analysis of immunogenicity data including sero-response rates (sero-protection rate and sero-conversion rate), and antibody titer.

\subsection{Early Phases of Clinical Development}

As any other biopharmaceutical product, a candidate vaccine, before market authorization, usually follows the three-phase (phases I, II and III) clinical development pathway after obtaining safety and immunogenicity evidence from preclinical studies such as in-vivo and in-vitro experiments. However, the urgent need for a safe and efficacious COVID-19 vaccine may mandate an expedited development route, in which regulatory filling may occur based on only limited data from early-phase trials. Because of this, a statistically efficient and scientifically rigorous early-phase design becomes critically important. This section describes some design features for phase I and phase II vaccine trials with special considerations in accelerated COVID-19 vaccine development for possibly early regulatory filing.

Phase I studies. The first-in-human administration of a candidate vaccine, often to a small number (say, 20-50) of immunocompetent naive healthy adults with low risk of infection of the target pathogen, is carried out to determine safety, reactogenicity, and immune response in an open-label, non-randomized manner (WHO, 2017). Reactogenicity refers to the property of a vaccine being able to induce some common, expected adverse reactions, especially excessive immunological responses and physical manifestation of inflammatory response after vaccination (Hervé et al., 2019), and can be further classified as systemic reactions (e.g., fever, malaise) and local reactions (e.g., tenderness, sore arm at injection site) (Hudgens et al., 2004). Safety analyses in phase I trials are primarily descriptive by reporting frequencies, proportions, grades, and severity of adverse events within certain period of time (e.g., 3-14 days post vaccination) or throughout the study. Some vaccine-related serious adverse events found in phase I trials may provide a clue for further investigation in later phase trials.

Phase I trials can be conducted in a step-down and -up manner by first enrolling healthy adults, then stepping-down to include adolescents and pediatric population, and stepping-up to enroll older adult volunteers. Enrolling older population in the trial can help better understand vaccine safety and immune response among older people who face a higher risk of complications from COVID-19 than younger individuals. For example, a phase I trial for LNP-encapsulated mRNA vaccine co-developed by Moderna and NIAID, originally designed to enroll 45 healthy adults aged between 18 and 55 years, now enrolls additional 60 older participants: 30 adults aged 56 to 70 years and 30 adults aged 71 years and older (clinicaltrials.gov, NCT04283461) (NIAID, 2020)

Often phase I trials also explore the dose-response relationship between different amounts of antigens representing different serotypes or virus strains and immune responses, the lowest amount of antigen that can elicit a protective immune response, routes of vaccine administration 
(e.g., injection or by mouth), time and vaccination schedules (time interval between primary dose and booster doses) (WHO, 2017). Immunological data generated from bioassays include (1) antigen-specific $\mathrm{T}$ cell response such as cluster of differentiation (CD) $4+$ and CD 8+ cytotoxic $\mathrm{T}$ lymphocytes and cytokines, (2) geometric mean concentration or geometric mean titers of antibodies, (3) seroconversion rate with pre-defined threshold, and (4) reverse cumulative distribution to show the proportion of vaccinees against antibody level (in logarithmic scale); see EMA (2005) and Nauta (2020) for details. Since phase I studies are the translation of potential findings from in-vivo and in-vitro studies into humans, the trial should be designed on a risk-based approach including risk identification, assessment and mitigation.

Another important area that is often thoroughly explored before and/or during phase I trials is vaccine quality, stability, $\mathrm{CMC}$ and manufacturing. Before applied to humans, a candidate vaccine should be well characterized for its potency of viral antigens as this is a critical factor in mediating vaccine-related toxicity and other adverse events. Specifications of potency should be set sufficiently narrow in order to precisely estimate starting doses. Candidate vaccines are often stored under various storage conditions (e.g., refrigerator, room temperature) for sufficient time to determine vaccine shelf-life. Manufacturing process should be described in great detail, including formulation, fermentation, biological structure of virus or subunit, and nucleic acid sequence (EMA, 2003; Goetz et al., 2010).

Phase II trials. Upon having satisfactory results in terms of safety and immunogenicity from a phase I study, the candidate vaccine proceeds to phase II clinical evaluation to further investigate safety and immunogenicity of the vaccine with the primary objective to optimize vaccine doses and schedule and gain as much information as possible for planning phase III trials. In addition, an immune correlate of protection may be further explored in a phase II trial, which can facilitate the interpretation of results in future clinical trials using the immune response as endpoints (EMA, 2005; WHO, 2017). The study population can be a subset of the intended target population to whom the vaccine may be administered upon approval, and may consist of hundreds up to thousands of adult individuals with high-risk exposure to SARS-CoV-2 (e.g., adult healthy subjects in epidemic regions), or frontline health professionals if vaccine safety can be reasonably guaranteed. In addition to standard inclusion and exclusion criteria, subject recruitment may also consider operational and medical factors that may preclude individuals from enrollment into the study (Farrington and Miller, 2001). Since a phase II trial is usually comparative in nature, subjects can be randomized and double-blinded to receive either the investigational vaccine (or one of its doses) or a placebo. Community-based randomization can be considered when a controlled trial is feasible and community-based information, e.g., population composition, disease pattern, different strains of virus, disease severity and pattern, is readily available (Singh and Mehta, 2016), which can further facilitate the trial design and analysis.

If multiple primary hypotheses are tested, multiplicity adjustment should be considered to control the probability of false findings (type I error) (Farrington and Miller, 2001). When a phase II trial is designed to obtain some preliminary information of clinical efficacy and/or monitor clinically important safety events, an appropriate design with a sufficient sample size can be chosen to fulfill this purpose.

As an example, a phase II trial for the adenovirus type-5 vector-based recombinant SARSCoV-2 vaccine (Ad5-nCoV) (ClinicalTrials.gov, NCT04341389), co-developed by CanSino Biological Inc. and Beijing Institute of Biotechnology, was designed as a randomized, doubleblinded and placebo-controlled clinical trial. The study comprises two dosage levels (middle-dose and low-dose) of the vaccine and one placebo arm and plans to enroll 500 healthy adults over 18 
years of age, with 250 subjects in the middle-dose arm, 125 subjects in the low-dose arm, and 125 subjects in the placebo arm. The primary objective of the trial is to evaluate the immunogenicity and safety of the Ad5-nCoV vaccine - which encodes for a full-length S protein of SARS-CoV-2 virus. The immunogenicity will be evaluated at days $0,14,28$ and 6 months after vaccination.

\subsection{Novel Trial Design to Expedite Clinical Development}

A large number of ongoing COVID-19 vaccine development programs worldwide suggests that novel trial designs can be considered in clinical development, such as a master protocol, to screen out candidate vaccines that are unsafe or ineffective and to continue assessment of promising vaccines based on early data. There are at least two advantages associated with master protocols: (1) the use of trial network with infrastructure in place to streamline trial logistics, improve data quality, and facilitate data collection and sharing, and (2) the use of a common protocol that incorporates innovative statistical approaches to trial design and analysis (Woodcock and LaVange, 2017). Master protocols are more often used in cancer drug studies for targeting and accelerating clinical development, and have attracted some interest in non-oncology fields, such as infection diseases (Dodd et al., 2016). Given the current COVID-19 pandemic situation, they may be even more appropriate to accelerate early-phase trials and/or mid- to late-phase trial conduct for vaccine registration; see WHO (2020b) for COVID-19 phase IIb/III vaccine trial synopsis using a master protocol with a common control arm under a sequential design.

A master protocol can be used to conduct the trial(s) for exploratory purposes or to support a marketing application and can be structured to evaluate, in parallel, different vaccines compared to their respective controls or to a single common control group (FDA, 2018). For COVID-19 vaccines, a master protocol can accommodate different types of candidate vaccines with specific safety features and hypotheses, different route of administration, and different doses. The protocol usually uses adaptive design strategies (more discussion in Section 4.4) with predefined criteria for dropping out or keeping candidate vaccines already in the trial or adding new candidate vaccines based on pre-specified endpoints which can be correlates of protection (e.g., antigen-specific antibodies) or laboratory-confirmed clinical disease of infection. The primary endpoint can be immune correlates of risk, pre-identified serious adverse events such as immunologically enhanced disease, correlates of protection, and laboratory confirmed clinical disease and/or its severity. Short-term vaccine efficacy can be assessed based on clinical disease of infection due to a short incubation time of SARS-CoV-2 infection. However, the long-term protection of a vaccine cannot be established as it requires data on long-lasting immune responses and protection against infection.

Since a master protocol generally involves multiple objectives, e.g., multiple candidate vaccines or doses, each of which corresponds to a hypothesis, multiplicity may occur due to multiple opportunities to make a positive claim on vaccine efficacy for a candidate vaccine. However, the impact of multiplicity depends on the type of master protocol, the trial design, and the research questions to be answered (Collignon et al., 2020). If a master protocol comprises multiple candidate vaccines with a common control group, multiplicity adjustment may be required to control the protocol-wise type I error rate (PWER). However, if a master protocol contains multiple candidate vaccines, each of which targets its own population with its own control group, no multiplicity adjustment is necessary because this trial design is similar to conducting separate, independent trials and decisions are made for individual candidate vaccines without referring to other vaccines. The degree of multiplicity adjustment depends on the research question. For early-phase screening trials using a master protocol, the PWER can be controlled at a higher- 
level, e.g., 0.1 or 0.15 , in order to allow for more candidate vaccines or doses to enter into the next phases of clinical development. However, for a confirmative trial with product registration purpose, the PWER should be controlled at level 0.05 (two-sided) with appropriately chosen multiplicity adjustment methods; see Dmitrienko and D'Agostino Sr (2017) for general discussion on statistical approaches to multiplicity issues in clinical trials.

\subsection{Vaccine Challenge Studies}

Protective effects of a vaccine can be evaluated by vaccine challenge studies in which vaccinated and unvaccinated individual can be compared after direct challenge with the target pathogen under controlled experimental conditions (Knight-Jones et al., 2014). Challenge studies often start from animal models for initial evaluation of human vaccines and then move to humans with effective vaccines. Potential benefits of human challenge trials may include, among others, proof of concept for candidate vaccines, clearer understanding of the pathogenesis of and immunity to a pathogen, and identification of potential correlates of protection (WHO, 2016). However, human challenge studies may not always be generalizable as the challenge population has always been healthy adults who may be very different from those at risk for natural disease. In addition, challenge trials are often designed to assess short-term protection (Shirley and McArthur, 2011).

Eyal et al. (2020) argue that human challenge trials of SARS-CoV-2 candidate vaccines could accelerate the clinical development and potential rollout of effective vaccines. Given the current pandemic situation of COVID-19, many people try to be self-isolated and a phase III trial may take a much longer time to have interpretable results. In this case, a human challenge trial may use much fewer volunteers to get meaningful results in a much shorter period of time (Callaway, 2020b). Eyal et al. (2020) outline a challenge study design that includes healthy volunteers from "previously uninfected individuals at relatively low risk of complications or mortality from SARS-CoV-2 infection... and who are at substantial risk of natural exposure to SARS-CoV-2." These healthy volunteers should be young adults with no chronic health conditions living in areas with high transmission rates. See WHO (2016) for more discussion on study design, operational aspects and ethical considerations of human vaccine challenge trials. A large scale, phase III trial can be conducted if a human challenge trial shows that a candidate vaccine is efficacious.

\section{Endpoints, Study Population, and Other Design Considera- tions in Late-Phase Trials}

Upon achieving satisfactory results on safety and immunogenicity (or clinical efficacy) from early phase trials, the candidate vaccine then moves into late-phase (phase III) development which is critical for regulatory registration and approval. Therefore, a phase III trial is usually designed with a sufficient sample size to test primary hypotheses regarding vaccine safety and efficacy. However, a seamless design combining phase II and phase III trials can be considered in the COVID-19 pandemic to speed up the vaccine development.

\subsection{Endpoints}

A hierarchy of multi-level objectives, such as primary, secondary and tertiary objectives, is usually specified in a phase III trial, and these objectives provide the basis for further defining endpoints to be studied. It is almost certain that the primary objectives pertain to the assessment of safety 
and efficacy of the investigational vaccine, which is usually formulated as primary hypotheses to be tested based on some suitably chosen primary endpoints.

Hierarchy of endpoints. Hudgens et al. (2004) present an array of endpoints (including disease incidence rate, virus infection rate, and immunological surrogate endpoints) for vaccine trials at various phases and discuss the rationale, advantages and disadvantages of each endpoint in different scenarios. From regulatory and public health perspectives, clinical disease with laboratory confirmation is perhaps an ideal endpoint for COVID-19 as it directly measures whether a vaccinee is protected from having the disease. Given that SARS-CoV-2 is highly contagious with multiple modes of transmission (WHO, 2020d), a short period of incubation time for disease manifestation after exposure (Lauer et al., 2020), and convenience of laboratory confirmation (CDC, 2020), a clinical disease is highly recommended as a primary endpoint to evaluate vaccine efficacy. Secondary endpoints may consist of (1) infection as a measure of protection from virus infection due to vaccination, and (2) serological endpoints including immunological surrogate endpoints such as antigen-specific antibodies, $\mathrm{T}$ cell response, etc., which can provide further exploration of the correlate of protection from having COVID-19. For SARS-CoV-2 virus, there are four structural proteins: the spike $(\mathrm{S})$, membrane $(\mathrm{M})$, envelope $(\mathrm{E})$, and nucleocapsid $(\mathrm{N})$ proteins, among which the spike protein appears to be the primary protein interacting with host cells and thus is likely to be the protein to which antibodies are raised, but this is not clear at this time (Phan, 2020).

Vaccine efficacy. Consider a two-arm randomized vaccine clinical trial. Let $\lambda_{\mathrm{V}}$ and $\lambda_{\mathrm{C}}$ denote the incidence risks of laboratory confirmed clinical disease for vaccine group and control group, respectively. Then, the vaccine efficacy (VE) is defined as, e.g., Halloran et al. (1997),

$$
\mathrm{VE}=1-\frac{\lambda_{\mathrm{V}}}{\lambda_{\mathrm{C}}}=1-\mathrm{RR},
$$

where $\mathrm{RR}=\lambda_{\mathrm{V}} / \lambda_{\mathrm{C}}$ denote the relative risk of the disease in the vaccine group relative to the control group. Then, VE in Equation (1) can be interpreted as the reduction in the number of cases with the infectious disease due to vaccination per unit case in the placebo group. Without taking into account censoring, the incidence risk $\lambda_{\mathrm{V}}$ can be approximated as $\hat{\lambda}_{\mathrm{V}}=n_{\mathrm{V}} / N_{\mathrm{V}}$, where $n_{\mathrm{V}}$ denotes the number of cases with the infectious disease (confirmed by both clinical manifestation of symptoms and laboratory test such as polymerase chain reaction (PCR) test for SARS-CoV-2 virus) and $N_{\mathrm{V}}$ the number of participants in the vaccine group (vaccinees), and $\lambda_{\mathrm{C}}$ can be approximated similarly. Note that Equation (1) can be used as a basis for testing the primary hypothesis with a pre-specified clinically (and epidemiologically) acceptable threshold on vaccine efficacy and therefore for sample size determination; see Section 4.5 and Heyse and Chan (2016).

Primary safety endpoint. If a serious safety concern (e.g., life-threatening adverse events) is pre-identified from preclinical and/or early clinical development, then an endpoint representing that safety concern (e.g., a single endpoint or a composite endpoint) should be taken into consideration in the design, monitoring and analysis of a phase III trial. Specifically, a sufficient sample size should be planned to demonstrate that the vaccine is safe or has acceptable safety (favorable benefit-risk) profile; see Section 4.5 for sample size determination and Section 6 for safety evaluation.

\subsection{Study Population}

Ideally the participants should be a random sample from the study population, defined by a set of inclusion and exclusion criteria, that represent the target population for whom the vaccine is 
intended to be administered upon approval. However, the study population can be chosen from high-risk populations, e.g., healthcare providers, frontline workers (e.g., delivery servicemen), and those working with potentially infected materials (e.g., medical wastes) if (1) a candidate vaccine shows promising efficacy results in early studies, (2) there is a high incidence rate of the disease and/or infection in unvaccinated population, and/or (3) there are unmet medical needs for those who desperately need a vaccine. Dean et al. (2019) point out that vaccine trials may target populations in the areas with highest geographic risk for disease transmission: (1) individuals and their household members who have direct contact with diagnosed cases, (2) populations in areas with geographic proximity to a new diagnosed case, and (3) individuals who are the first-, second-, or even third-generation contacts with a diagnosed case. Enrolling individuals from high-risk populations will not only provide as earlier protection as possible to the participants, but also likely require a smaller sample size to achieve the same statistical power to demonstrate vaccine efficacy.

Given the high-risk populations as the priority study population, the trial may consider recruiting participants who are at high-risk to develop severe illness, such as, older adults and people of any age who have serious underlying health conditions (e.g., chronic lung disease or moderate to severe asthma, serious heart conditions, diabetes), provided that the vaccine does not present any major serious safety issues that may complicate the underlying medical conditions to these participants (WHO, 2020e). With promising immunological and clinical outcomes of the vaccine, the trial can then consider recruiting low-risk adults, adolescents, pregnant women, pediatric and geriatric populations if the vaccine does not present any major safety concerns to these vulnerable populations. Extending to low-risk and vulnerable populations will ensure generalizability of vaccine efficacy and hence a broader public health value for protection against the infection.

\subsection{Randomization}

Randomization has traditionally been used as a gold standard in late-phase trials to evaluate efficacy of a new intervention as randomized, well-controlled trials would produce the most reliable and robust data on vaccine efficacy. There are a variety of randomization methods in clinical trials (Rosenberger and Lachin, 2015), some of which may be very useful in vaccine trials, especially under public health emergencies such as COVID-19. Kahn et al. (2018) provide a summary of randomization schemes for investigational vaccines during epidemics of emerging infectious diseases. These randomization methods fall into two basic categories: individual randomization and cluster randomization.

Individual randomization or individually randomized controlled trials (iRCT). Participants are individually randomized, according to predefined randomization ratio, to the investigational vaccine group or placebo group. In comparison with cluster randomization, iRCT design has at least two major advantages: (1) the measured and unmeasured baseline characteristics of participants can be better balanced between comparison groups, and (2) it generally requires a smaller sample size to show the same degree of vaccine efficacy with the same statistical power. The iRCT can be a one-stage randomization where individual participants are directly randomized to vaccine or placebo group, or a two-stage (stratified) randomization in which participants are first grouped into strata according to, say, clinical sites or neighborhood, and within each stratum (site or neighborhood), participants are then randomized to either vaccine or placebo group. Some drawbacks of iRCT design include logistic inconvenience when randomization is performed in small units, e.g., small community or household, and difficulty to keep blinding 
when the vaccine causes distinctive adverse effects (Nason, 2016).

Cluster randomized controlled trials (cRCT). In cRCT, clusters of participants are randomized as a unit to receive the investigational vaccine or placebo. A cluster may be defined as a medical center, hospital, school, or neighborhood, and may be chosen to match the anticipated vaccine delivery system, or defined as groups of individuals at high risk of infection such as frontline healthcare workers or based on their contacts (Campbell and Walters, 2014; Hayes and Moulton, 2017). Ideally, all members from participated clusters should be enrolled into the trial; however, a random sample of subjects from each cluster may also be acceptable if the sample is sufficiently representative of that cluster. The advantages of cRCT over iRCT include (1) cRCT is logistically convenient when clusters are well-defined and stable and (2) cRCT design can help estimate the direct and indirect vaccine effect on the reduction of incidence rate of the infectious disease (Hayes et al., 2000). However, there are several disadvantages of cRCT as compared with iRCT: (1) a larger sample size required by a cRCT than an iRCT due to intra-cluster correlation (e.g., similarity in population features and response to intervention and possible interaction among participants within cluster), leading to diminishing returns in power and precision as cluster size increases (Hemming et al., 2017), (2) a high probability of unbalanced baseline covariates between comparison groups resulted from cRCT due to fewer units (clusters) being randomized, and (3) possible contamination of participants due to movement and disease transmission (Hayes and Moulton, 2017). To reduce covariate imbalance, a small set of cluster-level matching variables (e.g., baseline prevalence rate, population demographics) can be used to stratify and match clusters, although this may increase operational complexity.

There are at least three types of cRCTs that can be considered in vaccine trials: (1) Parallel cRCT is the simplest cluster randomization in which clusters are randomized to either vaccine or placebo group and intervention does not change until the end of the trial. (2) Stepped wedge (or phased) cRCT design starts all clusters with placebo and gradually introduces the investigational vaccine to some clusters at a regular basis during the trial, until all clusters taking the vaccine by the end of the trial. It should be pointed out that the order in which the vaccine is introduced to individual clusters should be chosen at random and that care should be taken when estimating vaccine efficacy by comparing before- and after-vaccination for infectious disease with secular trends. (3) Ring vaccination design, a strategy of containing the spread of a disease by vaccinating only those who are at a high risk of infection, was used in the eradication of smallpox (Strassburg, 1982) and in the Ebola vaccine development program (Ebola ça Suffit Ring Vaccination Trial Consortium, 2015; Henao-Restrepo et al., 2017). A ring of cluster can be defined based on confirmed cases, representing people at risk of exposure to the pathogens, or geographically defined based on (first- or second-generation) contacts. Subjects within rings can be randomized as a cluster unit (cRCT) or individually in the ring of iRCT. Ring vaccination design is best suitable for an outbreak that is highly localized and vaccines that work quickly enough to protect the infection.

Some other randomization designs may also be considered for vaccine trials during public health emergencies, such as (1) two-stage randomization in which clusters are randomized to different levels of vaccine coverage and participants within clusters are then individually randomized to vaccine or placebo conditional on the vaccine coverage from the first randomization (WHO, 2020b) and (2) factorial trials that allow for simultaneous evaluation of multiple candidate vaccines, or multiple doses of the same candidate vaccine, and some other prevention interventions such as vector control of pathogens or behavioral risk reduction (Friedman et al., 2010, pp.264-270). 


\subsection{Adaptive Design}

In contrast to the conventional fixed-sample designs where the main aspects of a trial (e.g., sample size, randomization scheme, inclusion and exclusion criteria, vaccine doses) remain the same until trial completion, adaptive designs provide a flexibility of mid-course modification on trial procedures and/or statistical aspects. Examples of such modifications are an early termination for efficacy or futility, sample size re-estimation, changes in randomization scheme, etc., using accumulated data at interim analyses. These modifications must be prospectively defined in the study protocol to ensure scientific integrity of clinical research and are communicated with and accepted by regulatory agencies before trial begins.

In addition to the novel trial designs described in Section 3.4 for early phase studies, phase III trials can also be performed adaptively to incorporate cumulative information for early decisionmaking. Adaptive designs, now widely used in biopharmaceutical product development (Lin et al., 2016), are clearly advantageous over the conventional fixed-sample designs for COVID19 vaccine trials. First, knowledge about the biology of SARS-CoV-2 and the epidemiology of COVID-19 is limited, implying that a vaccine trial design needs to be adapted to the changing landscape (e.g., period of infectivity, immunogenicity, immunity, virus strains, etc.) as information accumulates. Second, given over 100 different candidate vaccines in development pipeline (Le et al., 2020; WHO, 2020c), sponsors may consider adaptive strategies (e.g., a seamless phase II/III clinical trials) to test multiple candidate vaccines (or doses) simultaneously and only the most promising candidate vaccines (or doses) can be advanced to the next phase of development; see also Section 3.4 for novel trial design to expedite clinical development. This type of adaptive strategies is particularly useful when a phase II trial enrolls only a few hundred individuals with varying dose, formulation and regimen. A seamless design can also be used for smooth and quick transition from early phase to late phase trials using appropriately selected surrogate markers for clinical protection. A successful example of the seamless phase II/III adaptive design was a trial for 9-valent human papilloma-virus (HPV) vaccine (HPV-9), in which participants are randomized to one of three HVP-9 doses or a comparator vaccine in a phase II trial. Then, the best vaccine dose was selected for phase III trial after the analysis of post-dose 2 immunogenicity and safety data (Chen et al., 2015; Lin et al., 2016). Third, adaptive design methods are commonly used for safety monitoring, especially serious safety concerns (see Section 6 for more discussion). Therefore, adaptive trial designs can accelerate vaccine development by rapidly screening out poor performing candidate vaccines while keeping the evaluation of promising ones, improving the characterization of more efficacious candidate vaccines and the identification of correlates of immune protection (Corey et al., 2011), which is critically important during the COVID-19 pandemic.

\subsection{Sample Size}

The sample size of a vaccine trial is driven by several major factors, e.g., primary objectives, primary endpoints, and trial design, among others. The primary objectives are usually expressed as primary hypotheses which may relate to vaccine efficacy (effectiveness) or safety or both. When a primary hypothesis is formulated on a safety endpoint e.g., a rare serious adverse event (AE) or an $\mathrm{AE}$ of special interest, the trail generally requires a much larger sample size due to a low incidence rate of the SAE. If a clinical endpoint (e.g., clinical infection) is chosen as the primary endpoint, the trial may also mandate a large sample size and/or longer follow-up time in order to observe sufficient infection cases to demonstrate vaccine efficacy. However, if 
a surrogate endpoint (e.g., antibodies) is used as a primary endpoint, which usually is the case for early-phase immunogenicity studies, a trial may require a smaller sample size. In contrast to the fixed-sample size design, adaptive designs (e.g., group sequential design) require a smaller sample size on average, but may lead to operational complexity (e.g., interim analyses, mid-course modification).

Consider a two-arm, fixed sample, individually randomized vaccine trial with laboratoryconfirmed clinical outcome of infection as a primary endpoint. Then, testing the primary hypothesis for vaccine efficacy can proceed in a variety of ways, depending on how the primary hypothesis is formulated, which could lead to different methods for sample size calculation. For example, the following sample size formulas correspond to testing vaccine efficacy in different scales:

1. Testing for equality of two Poisson rates: Assume the true Poisson rates $\lambda_{\mathrm{V}}$ for vaccine group and $\lambda_{\mathrm{C}}$ for control group and equal follow-up time $T$. To test $H_{0}: \lambda_{\mathrm{C}}-\lambda_{\mathrm{V}}=0$ against $H_{1}: \lambda_{\mathrm{C}}-\lambda_{\mathrm{V}}=\delta_{\lambda}>0$ at significant level $\alpha$ (two-sided) with $1-\beta$ power, the required amount of follow-up time (which can be translated into a sample size) for each group is (Hayes and Bennett, 1999)

$$
T_{\lambda}=\left(z_{1-\alpha / 2}+z_{1-\beta}\right)^{2} \frac{\lambda_{\mathrm{C}}+\lambda_{\mathrm{V}}}{\delta_{\lambda}^{2}}
$$

where $z_{a}$ is the $a$ th percentile of the standard normal distribution.

2. Testing for equality of two proportions: Let $\pi_{\mathrm{V}}$ and $\pi_{\mathrm{C}}$ denote the population incidence rates of infection for vaccine and control groups, respectively. To test $H_{0}: \pi_{\mathrm{C}}-\pi_{\mathrm{V}}=0$ against $H_{1}: \pi_{\mathrm{C}}-\pi_{\mathrm{V}}=\delta_{\pi}>0$, the required number of participants for each group is (Fleiss et al., 2013)

$$
n_{\pi}=\left(z_{1-\alpha / 2}+z_{1-\beta}\right)^{2} \frac{\pi_{\mathrm{C}}\left(1-\pi_{\mathrm{C}}\right)+\pi_{\mathrm{V}}\left(1-\pi_{\mathrm{V}}\right)}{\delta_{\pi}^{2}} .
$$

3. Testing for relative risk: Epidemiologists may be more willing to work on a relative scale such as relative risk. To test $H_{0}: \mathrm{RR}=R_{0}$ against $H_{1}: \mathrm{RR}=R_{1}<R_{0}$, where $\mathrm{RR}=\pi_{\mathrm{V}} / \pi_{\mathrm{C}}$ which is similar to that as defined in Equation (1), the required total sample size for both groups is given by (Blackwelder, 1993)

$$
N_{\mathrm{RR}}=\left(z_{1-\alpha / 2}+z_{1-\beta}\right)^{2} \frac{\left(1-\pi_{\mathrm{C}}\right) /\left(\kappa \pi_{\mathrm{C}}\right)+\left(1-\pi_{\mathrm{V}}\right) /\left[(1-\kappa) \pi_{\mathrm{V}}\right]}{\left[\log \left(R_{0}\right)-\log \left(R_{1}\right)\right]^{2}},
$$

where $\kappa=n_{\mathrm{C}} / N_{\mathrm{RR}}$ and $n_{\mathrm{C}}$ denotes the sample size in the control group.

Alternatively, one can assume that there are $x_{\mathrm{V}}$ infection cases in the vaccine group and $x_{\mathrm{C}}$ cases in the control group. Conditional on $X=X_{\mathrm{V}}+X_{\mathrm{C}}, X_{\mathrm{V}}$ is a binomial random variable with probability $p=\mathrm{RR} /(h+\mathrm{RR})$, where $h=(1-\kappa) / \kappa$. Then, testing $H_{0}: \mathrm{RR}=R_{0}$ against $H_{1}: \mathrm{RR}=R_{1}<R_{0}$ is equivalent to testing $H_{0}: p=p_{0}$ against $H_{1}: p=p_{1}<p_{0}$, where $p_{0}=\mathrm{RR}_{0} /\left(h+\mathrm{RR}_{0}\right)$ and $p_{1}=\mathrm{RR}_{1} /\left(h+\mathrm{RR}_{1}\right)$, which requires the total number of infection cases given by (Blackwelder, 1993)

$$
X=\frac{\left(z_{1-\alpha / 2} \sqrt{p_{0}\left(1-p_{0}\right)}+z_{1-\beta} \sqrt{p_{1}\left(1-p_{1}\right)}\right)^{2}}{\left(p_{0}-p_{1}\right)^{2}} .
$$

This translates into the total number of participants for both groups

$$
N=\frac{X}{\kappa \pi_{\mathrm{V}}+(1-\kappa) \pi_{\mathrm{C}}} .
$$


4. Confidence interval on vaccine efficacy. One can construct a $100(1-\alpha) \%$ confidence interval on vaccine efficacy and define a desired width of the confidence interval. Assuming equal numbers $n$ of participants in vaccine and control groups, then the required sample size is given by (O’Neill, 1988)

$$
n=\left(\frac{2 z_{1-\alpha}}{d}\right)^{2}\left(\frac{1+1 / \mathrm{RR}}{\lambda_{\mathrm{C}}}-2\right)
$$

where $d$ is the desired width of the confidence interval for $\mathrm{VE}$ and $\lambda_{\mathrm{C}}$ is as defined in Equation (2).

Sample size calculation methods in group sequential designs can be found, e.g., in Jennison and Turnbull (1999, Chapter 12) and Chow et al. (2017, Chapter 8). For example, consider a two-arm randomized trial with a group sequential design. Suppose that the interest is to test the null hypothesis $H_{0}: \mathrm{VE} \leq \mathrm{VE}_{0}$ against the alternative hypothesis $H_{1}: \mathrm{VE} \geq \mathrm{VE}_{1}$. Since VE is measured as the reduction of infection cases of the vaccinated group relative to the unvaccinated group, the number of infection cases in the unvaccinated (or vaccinated) group can be treated as a conditional binomial random variable, given the total number of observed infection cases from both groups. In other words, testing $H_{0}: \mathrm{VE} \leq \mathrm{VE}_{0}$ against $H_{1}: \mathrm{VE} \geq \mathrm{VE}_{1}$ is equivalent to testing $H_{0}: \pi \leq \pi_{0}=1 /\left(2-\mathrm{VE}_{0}\right)$ against $H_{1}: \pi \geq \pi_{1}=1 /\left(2-\mathrm{VE}_{1}\right)$, where $\pi=n_{\mathrm{U}} /\left(n_{\mathrm{V}}+n_{\mathrm{U}}\right), n_{\mathrm{V}}$ and $n_{\mathrm{U}}$ denote the numbers of infection cases from the vaccinated and unvaccinated groups, respectively. Using the algorithm described in Chapter 12 of Jennison and Turnbull (1999) and EAST ${ }^{\circledR} 6$ software, the sample sizes, O'Brian-Fleming-like boundaries for efficacy and futility, and boundary crossing probabilities under $H_{0}$ and $H_{1}$ of three sequential designs with six interim analyses and one final analysis are calculated for $\mathrm{VE}_{0}=0.3$ and $\mathrm{VE}_{1}=$ $\{0.55,0.60,0.65\}$ (Table 1). For example, if seven analyses are planned with the first interim analysis taking place at information fraction 0.4 , a total of 115 infection cases is needed to reject $H_{0}: \mathrm{VE}_{0}=0.30$ at significance level 0.025 (one-sided) and power 0.90 if the true $\mathrm{VE}_{1}=0.60$. The O'Brien-Fleming-like efficacy and futility bounds are expressed in terms of $n_{\mathrm{U}}$, the number of infection cases from the unvaccinated group. If the observed number of infection cases from unvaccinated group is equal to or greater than the upper bound for efficacy at any analysis, the vaccine can be declared efficacious (e.g., vaccine efficacy is at least at $\mathrm{VE}_{1}$ ); on the other hand, if the observed number of infection cases from unvaccinated group is less than the lower bound for futility at any analysis, the vaccine can be said non-efficacious (e.g., vaccine efficacy is no more than $\mathrm{VE}_{0}$ ) (Table 1).

There are more options for sample size determination. For example, Chan and Bohidar (1998) develop exact conditional and unconditional methods for power and sample size estimation; Farrington and Manning (1990) provide sample size formulas for comparing two binomial proportions with null hypothesis of non-zero risk difference or non-unity relative risk; Anderson et al. (2012) propose a two-stage adaptive design strategy with interim analyses to allow for evaluation of study feasibility and sample size adaptation for case-driven vaccine efficacy study when incidence rate is unknown; Hayes and Bennett (1999) describe sample size formulas for both unmatched and pair-matched cluster randomized trials. 
Table 1: Sample sizes, O'Brien-Fleming-like boundaries for efficacy and futility, and boundary crossing probabilities of sequential designs with seven analyses for testing $H_{0}: \mathrm{VE} \leq \mathrm{VE}_{0}$ against $H_{1}: \mathrm{VE} \geq \mathrm{VE}_{1}$ at significance level 0.025 (one-sided) and power 0.90 with Lan-DeMets spending functions for both type I and II errors.

\begin{tabular}{|c|c|c|c|c|c|c|c|c|}
\hline \multirow{2}{*}{$\begin{array}{l}\text { Look } \\
\#\end{array}$} & \multirow[b]{2}{*}{$I F^{1}$} & \multirow{2}{*}{$\begin{array}{l}\text { Total } \\
\text { Cases }\end{array}$} & \multirow{2}{*}{$\begin{array}{c}\mathrm{UBE}^{2} \\
\left(n_{\mathrm{U}} \geq\right)\end{array}$} & \multirow{2}{*}{$\begin{array}{c}\mathrm{LBF}^{3} \\
\left(n_{\mathrm{U}}<\right)\end{array}$} & \multicolumn{4}{|c|}{ Boundary Crossing Probability (Cumulative) ${ }^{4}$} \\
\hline & & & & & $P_{0}\left(\right.$ rej $\left.H_{0}\right)$ & $P_{0}\left(\operatorname{acc} H_{0}\right)$ & $P_{1}\left(\operatorname{acc} H_{1}\right)$ & $P_{1}\left(\right.$ rej $\left.H_{1}\right)$ \\
\hline \multicolumn{9}{|c|}{ Scenario $1 . \mathrm{VE}_{0}=0.30, \mathrm{VE}_{1}=0.65$, sample size 115 with $E_{0}(N)^{5}=60$ and $E_{1}(N)^{5}=78$} \\
\hline 1 & 0.400 & 46 & 39 & 27 & 0.000 & 0.498 & 0.080 & 0.009 \\
\hline 2 & 0.504 & 58 & 46 & 36 & 0.001 & 0.699 & 0.251 & 0.020 \\
\hline 3 & 0.600 & 69 & 52 & 44 & 0.003 & 0.821 & 0.448 & 0.033 \\
\hline 4 & 0.704 & 81 & 59 & 53 & 0.007 & 0.902 & 0.644 & 0.049 \\
\hline 5 & 0.800 & 92 & 66 & 61 & 0.012 & 0.944 & 0.776 & 0.065 \\
\hline 6 & 0.904 & 104 & 73 & 70 & 0.017 & 0.969 & 0.867 & 0.082 \\
\hline 7 & 1.000 & 115 & 79 & 79 & 0.021 & 0.979 & 0.903 & 0.098 \\
\hline
\end{tabular}

Scenario 2. $\mathrm{VE}_{0}=0.30, \mathrm{VE}_{1}=0.60$, sample size 172 with $E_{0}(N)=90$ and $E_{1}(N)=115$

$\begin{array}{rrrrrrrrr}1 & 0.401 & 69 & 55 & 40 & 0.000 & 0.487 & 0.091 & 0.009 \\ 2 & 0.500 & 86 & 65 & 53 & 0.001 & 0.682 & 0.255 & 0.020 \\ 3 & 0.599 & 103 & 75 & 65 & 0.003 & 0.814 & 0.456 & 0.033 \\ 4 & 0.698 & 120 & 85 & 77 & 0.007 & 0.895 & 0.637 & 0.048 \\ 5 & 0.802 & 138 & 95 & 90 & 0.012 & 0.944 & 0.779 & 0.065 \\ 6 & 0.901 & 155 & 105 & 102 & 0.017 & 0.969 & 0.862 & 0.082 \\ 7 & 1.000 & 172 & 115 & 115 & 0.021 & 0.980 & 0.900 & 0.099\end{array}$

Scenario 3. $\mathrm{VE}_{0}=0.30, \mathrm{VE}_{1}=0.55$, sample size 267 with $E_{0}(N)=140$ and $E_{1}(N)=177$

\begin{tabular}{rrrrrrrrr}
1 & 0.401 & 107 & 81 & 63 & 0.000 & 0.471 & 0.099 & 0.009 \\
2 & 0.502 & 134 & 96 & 81 & 0.001 & 0.673 & 0.271 & 0.020 \\
3 & 0.599 & 160 & 111 & 99 & 0.003 & 0.806 & 0.468 & 0.033 \\
4 & 0.700 & 187 & 127 & 118 & 0.007 & 0.891 & 0.649 & 0.049 \\
5 & 0.801 & 214 & 143 & 136 & 0.012 & 0.940 & 0.782 & 0.066 \\
6 & 0.899 & 240 & 158 & 155 & 0.017 & 0.966 & 0.863 & 0.082 \\
7 & 1.000 & 267 & 174 & 174 & 0.021 & 0.978 & 0.901 & 0.099 \\
\hline
\end{tabular}

${ }^{1} \mathrm{IF}$ : Information fraction in terms of the total number of infection cases from both groups.

${ }^{2} \mathrm{UBE}$ : Upper bound for efficacy in terms of $n_{\mathrm{U}}$, the number of infection cases from unvaccinated group.

${ }^{3} \mathrm{LBF}$ : Lower bound for futility in terms of $n_{\mathrm{U}}$, the number of infection cases from unvaccinated group.

${ }^{4} P_{0}\left(\right.$ rej $\left.H_{0}\right)$ denotes the probability of rejecting $H_{0}$ given $H_{0}$ is true, $P_{0}\left(\right.$ acc $\left.H_{0}\right)$ the probability of accepting $H_{0}$ given $H_{0}$ is true, $P_{1}\left(\operatorname{acc} H_{1}\right)$ the probability of accepting $H_{1}$ given $H_{1}$ is true, and $P_{1}\left(\right.$ rej $\left.H_{1}\right)$ the probability of rejecting $H_{1}$ given $H_{1}$ is true.

${ }^{5} E_{0}(N)$ denotes the expected sample size under $H_{0}$ and $E_{1}(N)$ the expected sample size under $H_{1}$.

\section{Assessing Vaccine Effectiveness Using Real-World Data and Evidence}

\subsection{Rationale for Using RWD \& RWE in Vaccine Trials}

Randomized controlled trials have many limitations, such as operational complexity, high costs, deviation from real-world medical practice, and lack of generalizability. In contrast, real-world studies are less expensive, logistically convenient, reflective of routine medical practice, and 
representative of general population, and therefore can be considered as an alternative to assess vaccine effectiveness (Tseng and Sy, 2018). Chen et al. (2020a) present rationales on whether real-world data and evidence (RWD \& RWE) can be used in the design and analysis of clinical trials for an investigational product with the following four considerations:

1. Scientific: Is the use of real-world data scientifically valid? What are potential challenges that may impact the scientific integrity of the trial? Can relevant assumptions regarding similarity between vaccinated and unvaccinated groups be reasonably verifiable? From scientific perspectives, using RWD \& RWE for vaccine efficacy evaluation may be a promising option, especially during the COVID-19 pandemic, partly because randomization sometimes may not be feasible, and blinding can be very difficult in some randomization schemes (e.g., cluster randomization with small cluster size). All of these may lead to operational difficulty of randomized trials.

2. Regulatory: Does the use of real-world data present potentially substantial challenges for regulatory decision-making or conflict with any regulatory guidelines? Sponsors are strongly recommended to have open communications with regulatory agencies on the use of real-world data and evidence before the design and conduct of any vaccine trials.

3. Ethical: What are the possible ethical issues if real-world data are not used in the design and analysis of vaccine trials? Does concurrent control group cause ethical problem in the trial? This may be the case in the COVID-19 pandemic if a vaccine is already shown to be fairly safe and efficacious in preventing SARS-CoV-2 infection.

4. Operational: What are the operational challenges in the design and analysis of clinical trials using real-world data? Is an independent statistician necessary to perform the design and analysis of the trial? How is the statistician blinded to outcome data when searching for a matched control? Yue et al. (2014) point out that it is critical to have a protection mechanism in place to prevent outcome information leaking, especially in the design phase, and such actions and specifications should be submitted to regulatory agencies for review and agreement.

See also FDA (2018) for a general RWE framework and NMPA (2020) for China National Medical Product Administration's regulatory guidance on the use of RWD \& RWE for medical product development and regulatory decision-making. A vaccine developer is highly encouraged to discuss with regulatory agencies regarding the above rationale of using RWD \& RWE in trial design and analysis for vaccine efficacy and effectiveness evaluation.

\subsection{Design Options Using RWD \& RWE}

Given the above rationale in mind, the vaccine developer may consider a vaccination program in a well-established healthcare system or community for an investigational COVID-19 vaccine that has been demonstrated to be safe and immunogenically responsive in early phase trials. The candidate vaccine can be given to a group of participants in the healthcare system or a community, without randomization and a pre-defined placebo group, and vaccine effectiveness can be evaluated by examining reported infection cases in the electronic health records of participants in the same source population. A simple design in vaccine effectiveness assessment is the case-control design in which a case can be chosen from the study population and one or more controls are randomly selected, or matched based on some pre-defined variables, from the same population. Some variations of case-control design include case-control design and test-negative design. In the case-control design, the controls are sampled either at the start or the end of the observational period and a control (or case) who is sampled at the start may become a case (or 
a control) at the end of follow-up. The test-negative design identifies cases and controls from those who seek healthcare services for COVID-19 related illness, e.g., fever, cough, and shortness of breath. It has the advantage of eliminating confounding due to healthcare seeking behavior. The test-negative design, in which patients who test positive are the cases and those who test negative are the controls, requires a highly specific test to reduce bias in estimated vaccine effectiveness and an assumption that the vaccine does not provide cross-protection to other diseases with similar symptoms (Ohmit et al., 2014; Nauta, 2020)

\subsection{Challenges When Using RWD \& RWE in Vaccine Trials}

There are some challenges in assessing vaccine effectiveness using RWD. First, individuals who are vaccinated may be different from those who are not in terms of their baseline characteristics, e.g., vaccinees may have better healthcare access and healthcare awareness than those who are not vaccinated and some healthcare providers may be more likely to recommend the vaccine than others. This type of imbalance in participant's characteristics is often called confounding which, if not adjusted, may cause confounding bias in the estimate of vaccine effectiveness. To overcome potential confounding bias, a commonly used approach is to match the case with one or more controls based on a set of key baseline covariates. Matching can be performed in a variety of ways, such as nearest neighbor matching and propensity score based subclassification. Another commonly used method removing confounding bias is inverse probability of treatment weighting that creates a "pseudo-population" in which the treatment is independent of the measured confounders; see Chen et al. (2020a) for an overview of matching and other statistical methods in using RWD \& RWE to inform trial design and analysis.

Second, there may be variations in the criteria and practice of case adjudication, which can lead to inconsistency of case definition and diagnosis and hence have an implication in the estimated vaccine effectiveness. There are currently at least two different types of diagnostic tests available for testing the infection of SARS-CoV-2 - PCR test for detecting viral RNA and antibody test for prior viral infection, e.g., Petherick (2020); see also https://www.who.int/ csr/sars/labmethods/en/ - and many testing kits for each of these two diagnostic tools are produced by different manufacturers and approved for use by different authorities across the world. Therefore, a high degree of sensitivity and specificity of the diagnostic tests is desired and a standard operating procedure to conduct the test should be in place.

Third, if electronic health records are used for identification of cases and controls, extra effort may be needed to ensure that vaccine exposure, infection (outcome), and some key covariates that are associated with vaccine access, vaccination preference, healthcare behavior, etc., are available in the databases. Some of these variables may require retrospectively tracing back for reliability and validity; see Levenson et al. (2020, Section 6) for an overview and a general discussion on outcome and study variable in real-world studies. Cases with confirmed infection may be underestimated if patients with minor symptoms don't go to clinics or hospitals for diagnostic test, which may lead to a biased sample for the infected cases and hence incorrect estimate of vaccine effectiveness.

In summary, a clinical trial using real-world data and evidence to evaluate vaccine effectiveness has the potential to accelerate clinical development, to efficiently use existing healthcare and/or vaccine delivery systems, to possibly benefit more participants with an efficacious vaccine, and to save development time and costs. On the other hand, the trial should be designed carefully to handle some known (and unknown) confounding variables in order to correctly estimate vaccine effectiveness with minimum bias. 


\section{Vaccine Safety}

\subsection{A General Strategy for Safety Evaluation}

As discussed in Section 2, preventive vaccines are administered to healthy populations and therefore usually have a very high standard in safety. Safety information starts accumulating from the in-vivo and in-vitro laboratory testing to early- and late-phase clinical trials. Different types of vaccines may have very different safety features, which can also be impacted by their manufacturing processes.

Some adverse events may be common, expected, and less serious, such as those related to immunological responses and injection-site reactions as discussed in Section 3.3. For these adverse events, a general approach in the design stage is to specify some statistical methods for summarization, analysis, and graphical display of safety data (e.g., the difference in incidence rates between vaccinated and unvaccinated groups for individual safety events), and to design a set of statistical and graphical tools for use in routine safety monitoring by the Data and Safety Monitoring Committee during the course of vaccine trial; see Chen et al. (2018) for more detail description on the methods and tools.

Perhaps a major concern for SARS-CoV-2 vaccines is some rare serious adverse events (SAE), which may be biologically plausible. Examples of such serious adverse events are the so-called cell-based enhancement (CBE) and antibody-dependent enhancement (ADE) (Negro, 2020; Peeples, 2020). CBE is a category of events that includes allergic inflammation caused by Th2 immunopathology, an undesired immune reaction induced by type 2 T-cell helpers. In previous studies of SARS, aged mice were found to have high risks of having life-threatening Th2 immunopathology (Bolles et al., 2011). ADE is a well-known group of events for which people who get vaccinated have a higher risk of developing a more severe form of the disease than those who don't get vaccinated. For example, vaccinated ferrets with a recombinant modified SARS vaccine developed inflammatory liver damage (enhanced hepatitis) (Weingartl et al., 2004). For component vaccines using $\mathrm{S}$ protein against SARS-CoV-2, it was found that the full-length $\mathrm{S}$ protein can cause severe liver damage and may result in enhanced infection (Du et al., 2009), which may be caused by $\mathrm{S}$ protein specific antibodies. However, it is unclear which domains and amino acids in the $\mathrm{S}$ protein of SARS-CoV-2 are involved in liver damage. Therefore, COVID-19 vaccines using full-length $\mathrm{S}$ protein as an antigen may face more safety challenges and hence require a carefully designed safety monitoring and evaluation strategy during clinical development and post-approval surveillance.

\subsection{A Major Safety Endpoint in a Primary Hypothesis}

For SAEs with regulatory and public health importance, the sponsor may consider incorporate these type of events into the primary objective and hypotheses and then determine appropriate sample size to demonstrate whether the vaccine has an acceptable safety profile (e.g., relative risk of the vaccinated in comparison with unvaccinated to develop the event is less than a threshold) or is unsafe (e.g., the relative risk is greater than the threshold). The threshold on relative risk (or any other risk scales) for acceptable safety profile has to be discussed with and agreed by regulatory agencies, and needs to take into account the severity and incidence rate of the SAE and its impact on public health. An excellent example using a major safety event in the design of an vaccine trial is the Rotavirus Efficacy and Safety Trial (REST) which uses a group sequential design, with a maximum sample size of 100,000 and a minimum of 60,000 infants with complete follow-up, to detect a 10-fold increase in relative risk $(\mathrm{RR})$ of intussusception in vaccinees during 
the 42-day follow-up period after vaccination (Heyse et al., 2008). There are several options in statistical methodologies for deriving sequential monitoring boundaries, such as the sequential generalized likelihood ratio (GLR) test (Shih et al., 2010) and maximized probability ratio test (maxSPRT) (Kulldorff et al., 2011), with the latter being a special case of the sequential GLR. Detail discussions and application of sequential GLR or maxSPRT in setting up safety monitoring boundaries can be found in Shih et al. (2010); Chen (2014) and Chen et al. (2018).

\subsection{Pharmacovigilance and Risk Management}

A pharmacovigilance and risk management plan (PRMP) is essential before the conduct of any vaccine trials, and this is perhaps even more important for accelerated vaccine development due to public health urgency such as COVID-19 vaccines. A vaccine PRMP relates to detection, assessment, understanding, management, mitigation, and communication of adverse events following vaccination, and to the prevention of untoward effects of the vaccine. The practical aspects of a vaccine PRMP include (1) establishment of a PRMP committee and its roles and responsibilities, (2) data capture, reporting, and coding, (3) event adjudication processes, (4) active surveillance of specific adverse events of interest and general safety endpoints, (5) application of statistical tools for signaling, (6) risk management and mitigation, and (7) risk communication. The analysis of safety events generally follows the three-tier algorithm of Gould (2003) based on the premise that it is important to report all adverse events, but not all events need to be analyzed in the same way; see Crowe et al. (2009) and Chuang-Stein and Xia (2013) for more discussions from practical perspective.

The breadth and depth of vaccine safety assessment mandate a multi-disciplinary approach involving experts with different professional backgrounds (basic science, clinical science, regulatory science, data science, statistics and epidemiology) to work together for planning and execution of a safety evaluation program, and for interpretation and communication of safety concerns in a timely manner. It also traverses industry, government, academia and the public. A general principle and applications for vaccine pharmacovigilance can be found in (CIOMS, 2012).

\section{Other Considerations}

This section discusses some points that may not be immediately applicable to COVID-19 vaccine development and regulatory filing, but can be considered for an evaluation of long-lasting vaccine efficacy which could be part of the development plan. In fact, statistical modeling and simulation can use data generated from early-phase trials and/or short-followup studies to help evaluate the immune correlates of protection, herd immunity, vaccine direct and indirect effects, and waning vaccine efficacy.

\subsection{Correlates of Protection}

Correlates of protection refers to surrogates, often immune responses, that can help predict protection against infection or reduce the severity of the disease after infection of a pathogen. In clinical development of a vaccine product as described in Section 3, immune responses are primary outcome variables for early-phase studies and clinical outcomes such as clinical disease or infection are used to measure vaccine efficacy and effectiveness in late-phase trials and realworld studies. Then, some questions naturally arise: How the immune responses as correlates 
of protection can help make a go/no go decision from an early-phase study to a late-phase trial and to more precisely predict clinical outcome? What level or threshold of the correlates is sufficient to provide protection against infection or reduce the severity of disease? Are the correlates broader enough to provide protection against different strains of virus? Correlates of protection are valuable markers in vaccine trials as they can help (1) understand the mechanism of protection, (2) identify promising candidate vaccines, (3) use early-phase and/or short followup immunogenicity data to plan late-phase trials, (4) provide endpoints for further evaluation of vaccine trials by regulatory and public health authorities (Dudasova et al., 2020).

To evaluate a surrogate, Prentice (1989) proposes four criteria to validate whether the surrogate can be used as a substitute for clinical outcome, which can also be adapted for vaccine trials. To determine a threshold of correlates for protection, regression models such as logistic regression can be used to establish an appropriate threshold above which a reasonable level of protection against infection in terms of clinical outcome can be expected (Nauta, 2020, pp. 164-172). With limited data from early-phase trials on immunogenicity and clinical outcome of interest, extensive modeling and simulation may help characterize and establish correlates of protection, provide early indication for go/no go decision, and hence accelerate vaccine clinical development. See also WHO (2013) for some other considerations in the validation of correlates of protection in vaccine trials. Recently, Dudasova et al. (2020) propose a new framework, called PoDBA: Probability of Disease Bayesian Analysis, that uses a three-parameter sigmoid function to model the relationship between the probability of disease and immune response biomarker values (in $\log$ scale) that are collected shortly after vaccination. They use PoDBA to estimate vaccine efficacy and its confidence interval based on probability of disease curve and biomarker distributions in vaccinated and unvaccinated groups. The approach outperforms the standard estimation method in terms of accuracy and precision, as demonstrated by stochastic simulations, and uses much less data than in a full phase III trial. This property of PoDBA (higher precision per subject studied) is generally expected to hold as long as the measured immunogenicity marker (and any key covariates) contains sufficient information relevant to the vaccine's protective immunogenicity, i.e. the correct (predictive) assay is being used. Applications of this method to three difference vaccine datasets yields consistent results with standard estimate of vaccine efficacy (Dudasova et al., 2020).

Regarding immune responses as correlates of protection for COVID-19, Bao et al. (2020) present a study of two rhesus macaques who had recovered from SARS-CoV-2 infection with mild illness and did not seem to be re-infected when rechallenged with the virus. Lurie et al. (2020) point out that even though "correlates of protection may be inferred from experience with SARS and MERS vaccines, they are not yet established. As with naturally acquired infection, the potential duration of immunity is unknown; similarly, whether single-dose vaccines will confer immunity is uncertain." Like many other SARS-CoV-2 vaccines in development, the NIH-Moderna sponsored phase I trial of an investigational vaccine for COVID-19 which consists of an RNA molecule that is designed to train the body immune system to generate antibodies that can recognize and intercept the spike protein that the virus uses to attack human body. A successful SARS-CoV-2 vaccine will need to prompt the human body to generate antibodies that block other viral proteins or make $\mathrm{T}$ cells that can recognize and kill infected cells (Callaway, 2020a). 


\subsection{Natural Herd Immunity and Direct and Indirect Vaccine Effect}

Herd immunity refers to the totality of naturally acquired and vaccine-induced immunity to a given pathogen in a population, usually defined by geographic regions. During the pandemic of COVID-19, multiple sources of data (Bai et al., 2020; Mizumoto et al., 2020; Nishiura et al., 2020) indicate that as high as $25 \%-50 \%$ of SARS-CoV-2 infected individuals remain asymptomatic and most likely undiagnosed. These undiagnosed individuals, plus those who are confirmed infection cases,can produce SARS-CoV-2 specific antibodies which may boost the background natural immunity against infection in some populations and may possibly impact the proportion of severe form of COVID-19 after re-infection with virus strains other than the first infection. Therefore, one may need to take into account the degree of naturally acquired herd immunity in the study population to ensure a sufficient sample size for demonstrating vaccine efficacy in a vaccine trial.

In vaccine effectiveness studies using cluster randomization, real-world data and/or observational studies, one may be able to assess the direct and indirect vaccine effects. Halloran et al. (1991) define the direct effect as the expected difference between the outcome of an individual receiving the vaccine and the potential outcome the same individual would have experienced had he/she not been given the vaccine. An example of direct effect is the reduction in the probability of being infected that results from being vaccinated, given an exposure to infection. The indirect effect of a vaccination program on an individual is the expected difference between the outcome of an individual who are not vaccinated in a community with vaccination program and the outcome of an individual who are not vaccinated in a community without vaccination program. Therefore, the level of herd immunity, either naturally acquired or vaccine-induced, should be taken into account in vaccine trial design when using direct and indirect effects to measure vaccine effectiveness.

\subsection{Waning Vaccine Efficacy}

Long-term protection against infection has been achieved for some vaccines, immunity wanes over time for some others due to, e.g., declining immunologic memory and antigenic variations of the pathogen (Hudgens et al., 2004; Lipsitch, 2019). Examples of waning efficacy of vaccines for which evolution of pre-dominant serotypes lead to diminishing vaccine efficacy include pertussis vaccines (Klein et al., 2012) and influenza vaccines (Ray et al., 2019). Although novel technologies in vaccinology, e.g., adjuvants, delivery system, antigen formulation and fermentation, have the potential to elicit more durable immune responses and hence possibly provide long-term protection (Gu et al., 2017), conflicting evidence shows that antibodies against the Middle East respiratory syndrome (MERS) virus, one of coronaviruses, drop precipitously for those who recover from MERS while antibodies against SARS virus are still present in the body 15 years after infection. Therefore, there is no solid evidence on whether the immune response is sufficient to prevent reinfection, nor good data showing long-lasting immunity for both SARS and MERS (Callaway, 2020a).

Studying vaccine-induced waning immunity not only provides more accurate prediction of protection against infection over time, but also has a great impact on vaccinology including boosting scheduling, choice of adjuvants, manufacturing technology and processes, and immunization routes in order to enhance long-term protective immunity. One of the recommendations from Waning Immunity and Microbial Vaccines Workshop by the National Institute of Allergy and Infectious Diseases is to apply modern immunologic tools to describe the correlates of immunity for vaccines that induce durable immunity (Gu et al., 2017), which can be incorporated into trial 
design and analysis through statistical modeling and simulation of waning vaccine efficacy over time. Specifically, one can apply nonparametric smooth functions such as those in Durham et al. (1999) or the likelihood-based approach in Kanaan and Farrington (2002) that incorporates a variety of assumptions for efficacy model, ascertainment bias and age dependence of infection hazard for estimating time-varying vaccine efficacy; see also Halloran et al. (2010, pp. 143-149) for technical details and application examples.

\section{Conclusion and Recommendations}

Vaccine perhaps is the best solution for protection against SARS-CoV-2 infection or reinfection. Vaccine development demands full understanding of SARS-CoV-2 virus (e.g., biological and genomic structure, pathogenesis, immunogenicity) and human body responses (e.g., cellular and humoral immune responses to virus infection), both are essential for vaccine design. Although this process takes time as scientific research has its own rule of timeline, scientists race against time in searching candidate vaccines that are safe and effective, during which statistical considerations are essential to help the vaccine development at pandemic speed. This paper gives a brief background on the immunology of vaccination against infection and presents some key design considerations for vaccine clinical trials from statistical perspective with a focus on phase III vaccine trials.

Given the current pandemic paradigm, a safe and effective COVID-19 vaccine is in paramount demand. With all the key points discussed in this paper, it is highly recommended that (1) novel trial design (e.g., master protocol) can be used in early-phase trial to screen out candidate vaccines due to safety concerns or futility and continue evaluation of promising vaccines, (2) human challenge trials can be performed to accelerate clinical development, (3) adaptive strategies (e.g., group sequential design, seamless phase II/III design, keep-the-winner or drop-the-loser design with multiple candidate vaccines or doses) be considered to expedite the development, (4) extensive modeling and simulation be used to establish reliable correlates of protection, threshold, and waning efficacy, (5) safety endpoints (e.g., enhanced disease, or any other vaccine-induced immunological disease) be thoroughly investigated, both clinically and statistical (some serious adverse events can be used in designing large scale trials), (6) real-world data and evidence can be considered under certain circumstances for vaccine efficacy and effectiveness evaluation, and (7) global collaboration and integration of vaccine development programs, e.g., Cattani (2020), be initiated to more efficiently use resource, expertise, and trial data (e.g., multiple candidate vaccines from different developers can form a joint development program, for which an efficient trial design can be used). Finally, open communication with regulatory agencies on trial design is highly recommended to ensure scientific integrity of clinical research and compliance of trial conduct with regulation and to expedite review and approval process.

\section{Acknowledgements}

The authors would like to thank Keaven Anderson, Ray Bain, Richard Baumgartner, Jessica Y. Chen, Jonathan Hartzel, and Lisa Lupinacci for their constructive comments that help improve the presentation and readability of the paper. Our special thanks go to Nick Kartsonis and Jeffrey R. Sachs who provide insightful, update-to-dated vaccine development process that helps greatly improve the accuracy and precision of the information presented in this paper. We are also grateful of the Editor and referee for their speedy review. 


\section{References}

Anderson KM, Chan IS, Li X (2012). An adaptive design for case-driven vaccine efficacy study when incidence rate is unknown. Statistics and Its Interface, 5(4): 391-399.

Bai Y, Yao L, Wei T, Tian F, Jin DY, Chen L, et al. (2020). Presumed asymptomatic carrier transmission of COVID-19. JAMA, 323(14): 1406-1407.

Bao L, Deng W, Gao H, Xiao C, Liu J, Xue J, et al. (2020). Reinfection could not occur in SARS-CoV-2 infected rhesus macaques. BioRxiv preprint: https://doi.org/10.1101/2020. 03.13 .990226$.

Blackwelder WC (1993). Sample size and power for prospective analysis of relative risk. Statistics in Medicine, 12(7): 691-698.

Bolles M, Deming D, Long K, Agnihothram S, Whitmore A, Ferris M, et al. (2011). A doubleinactivated severe acute respiratory syndrome coronavirus vaccine provides incomplete protection in mice and induces increased eosinophilic proinflammatory pulmonary response upon challenge. Journal of Virology, 85(23): 12201-12215.

Callaway E (2020a). Coronavirus vaccines: Five key questions as trials begin. Nature, 579: 481.

Callaway E (2020b). Should scientists infect healthy people with the coronavirus to test vaccines? Nature, 580(7801): 17.

Campbell MJ, Walters SJ (2014). How to Design, Analyse and Report Cluster Randomised Trials in Medicine and Health Related Research. John Wiley \& Sons, Hoboken, New Jersey.

Cattani M (2020). Global coalition to accelerate COVID-19 clinical research in resource-limited settings. Lancet, 395: 1322-1325. DOI: https://doi.org/10.1016/S0140-6736(20)30798-4.

CDC (2020). CDC tests for COVID-19. Centers for Disease Control and Prevention (https: //www.cdc.gov/coronavirus/2019-ncov/about/testing.html).

Chan IS, Bohidar NR (1998). Exact power and sample size for vaccine efficacy studies. Communications in Statistics-Theory and Methods, 27(6): 1305-1322.

Chen J (2014). Sequential testing for safety evaluation. In: Statistical Methods for Evaluating Safety in Medical Product Development (LA Gould, ed.), 319-331. Wiley Online Library.

Chen J, Heyse J, Lai TL (2018). Medical Product Safety Evaluation: Biological Models and Statistical Methods. Chapman \& Hall/CRC Press, Boca Raton, Florida.

Chen J, Heyse JF, Heaton P, Kuter BJ (2010). Age dependence of the risk of intussusception following tetravalent rhesus-human reassortant rotavirus tetravalent vaccine: Is it beyond doubt? American Journal of Epidemiology, 171(9): 1046-1054.

Chen J, Ho M, Lee K, Song Y, Fang Y, He W, et al. (2020a). The current landscape in biostatistics of real-world data and evidence: Use of RWD/RWE to inform clinical study design and analysis.

Chen WH, Strych U, Hotez PJ, Bottazzi ME (2020b). The SARS-CoV-2 vaccine pipeline: An overview. Current Tropical Medicine Reports. Forthcoming, https://doi.org/10.1007/ s40475-020-00201-6.

Chen YJ, Gesser R, Luxembourg A (2015). A seamless phase IIB/III adaptive outcome trial: Design rationale and implementation challenges. Clinical Trials, 12(1): 84-90.

Chow SC, Shao J, Wang H, Lokhnygina Y (2017). Sample Size Calculations in Clinical Research. Chapman and Hall/CRC, Boca Raton, Florida.

Chuang-Stein C, Xia HA (2013). The practice of pre-marketing safety assessment in drug development. Journal of Biopharmaceutical Statistics, 23(1): 3-25.

CIOMS (2012). Definition and application of terms for vaccine pharmacovigilance: Report of CIOMS/WHO working group on vaccine pharmacovigilance. Geneva: Council 
for International Organizations of Medical Sciences (https://cioms.ch/working_groups/ vaccine-pharmacovigilance/).

Clem AS (2011). Fundamentals of vaccine immunology. Journal of Global Infectious Diseases, $3(1): 73-78$.

Collignon O, Gartner C, Haidich AB, James Hemmings R, Hofner B, Pétavy F, et al. (2020). Current statistical considerations and regulatory perspectives on the planning of confirmatory basket, umbrella, and platform trials. Clinical Pharmacology \& Therapeutics, 107(5): 10591067.

Corey L, Nabel GJ, Dieffenbach C, Gilbert P, Haynes BF, Johnston M, et al. (2011). HIV-1 vaccines and adaptive trial designs. Science Translational Medicine, 3(79): 79ps13.

Crowe B, Xia H, Berlin J, Watson D, Shi H, Lin S, et al. (2009). Recommendations for the safety planning, data collection, evaluation and reporting during drug, biologic and vaccine development: A report of the safety planning, evaluation and reporting team. Clinical Trials, 6: $430-40$.

Cunningham AL, Garçon N, Leo O, Friedland LR, Strugnell R, Laupèze B, et al. (2016). Vaccine development: From concept to early clinical testing. Vaccine, 34(52): 6655-6664.

Dean NE, Gsell PS, Brookmeyer R, De Gruttola V, Donnelly CA, Halloran ME, et al. (2019). Design of vaccine efficacy trials during public health emergencies. Science Translational Medicine, 11(499): eaat0360.

Dmitrienko A, D'Agostino Sr RB (2017). Multiplicity issues in clinical trials. Statistics in Medicine, 36(28): 4423-4426.

Dodd LE, Proschan MA, Neuhaus J, Koopmeiners JS, Neaton J, Beigel JD, et al. (2016). Design of a randomized controlled trial for Ebola virus disease medical countermeasures: PREVAIL II, the Ebola MCM study. The Journal of Infectious Diseases, 213(12): 1906-1913.

Du L, He Y, Zhou Y, Liu S, Zheng BJ, Jiang S (2009). The spike protein of SARS-CoV-a target for vaccine and therapeutic development. Nature Reviews Microbiology, 7(3): 226-236.

Dudasova J, Laube R, Valiathan C, Wiener M, Gheyas F, Fiser P, et al. (2020). PoDBA: A method to estimate probability of disease and vaccine efficacy from clinical trial immunogenicity data. (To be submitted).

Durham SR, Walker SM, Varga EM, Jacobson MR, O'Brien F, Noble W, et al. (1999). Longterm clinical efficacy of grass-pollen immunotherapy. New England Journal of Medicine, 341(7): 468-475.

Ebola ça Suffit Ring Vaccination Trial Consortium (2015). The ring vaccination trial: A novel cluster randomised controlled trial design to evaluate vaccine efficacy and effectiveness during outbreaks, with special reference to Ebola. BMJ, 351: h3740.

Ellenberg SS, Keusch GT, Babiker AG, Edwards KM, Lewis RJ, Lundgren JD, et al. (2018). Rigorous clinical trial design in public health emergencies is essential. Clinical Infectious Diseases, 66(9): 1467-1469.

EMA (2003). Guideline on comparability of medicinal products containing biotechnology-derived proteins as active substance. Non-clinical and clinical issues. European Medicines Agency: Committee for Proprietary Medicinal Products.

EMA (2005). Guideline on clinical evaluation of new vaccines. European Medicines Agency. EMEA/CHMP/VWP/164653/2005, 2006 (https://www.ema.europa.eu/en/documents/ scientific-guideline/guideline-clinical-evaluation-new-vaccines_en.pdf).

Enjuanes L, Zuñiga S, Castaño-Rodriguez C, Gutierrez-Alvarez J, Canton J, Sola I (2016). Molecular basis of coronavirus virulence and vaccine development. In: Advances in Virus Research 
(J Ziebuhr, ed.), volume 96, 245-286. Elsevier.

Eyal N, Lipsitch M, Smith PG (2020). Human challenge studies to accelerate coronavirus vaccine licensure. The Journal of Infectious Diseases, 221(11): 1752-1756.

Farrington C, Miller E (2001). Vaccine trials. Molecular Biotechnology, 17(1): 43-58.

Farrington CP, Manning G (1990). Test statistics and sample size formulae for comparative binomial trials with null hypothesis of non-zero risk difference or non-unity relative risk. Statistics in Medicine, 9(12): 1447-1454.

FDA (2015). Analytical procedures and methods validation for drugs and biologics. Food and Drug Administration, US Department of Health and Human Services (https://www.fda.gov/ media/87801/download).

FDA (2018). US Food and Drug Administration, Silver Spring, MD (https://www.fda.gov/ media/120060/download).

FDA (2018). Master protocols: Efficient clinical trial design strategies to expedite development of oncology drugs and biologics. guidance for industry. Food and Drug Administration (https: //www.fda.gov/media/120721/download).

Fleiss JL, Levin B, Paik MC (2013). Statistical Methods for Rates and Proportions. John Wiley \& Sons, New York.

Friedman LM, Furberg C, DeMets DL, Reboussin DM, Granger CB (2010). Fundamentals of Clinical Trials, volume 4. Springer, New York.

Gauger PC, Vincent AL (2020). Serum virus neutralization assay for detection and quantitation of serum neutralizing antibodies to Influenza A virus in swine. In: Animal Influenza Virus Methods and Protocols (E Spackman, ed.), 321-333. Springer, 3rd edition.

Goetz KB, Pfleiderer M, Schneider CK (2010). First-in-human clinical trials with vaccines-what regulators want. Nature Biotechnology, 28(9): 910-916.

Gould A (2003). Practical pharmacovigilance analysis strategies. Pharmacoepidemiology and Drug Safety, 12(7): 559-574.

Gu XX, Plotkin SA, Edwards KM, Sette A, Mills KH, Levy O, et al. (2017). Waning immunity and microbial vaccines - workshop of the national institute of allergy and infectious diseases. Clinical and Vaccine Immunology, 24(7): e00034-17.

Halloran ME, Haber M, Longini Jr IM, Struchiner CJ (1991). Direct and indirect effects in vaccine efficacy and effectiveness. American Journal of Epidemiology, 133(4): 323-331.

Halloran ME, Longini IM, Struchiner CJ, Longini IM (2010). Design and Analysis of Vaccine Studies, volume 18. Springer, New York.

Halloran ME, Struchiner CJ, Longini Jr IM (1997). Study designs for evaluating different efficacy and effectiveness aspects of vaccines. American Journal of Epidemiology, 146(10): 789-803.

Hayes R, Alexander ND, Bennett S, Cousens S (2000). Design and analysis issues in clusterrandomized trials of interventions against infectious diseases. Statistical Methods in Medical Research, 9(2): 95-116.

Hayes R, Bennett S (1999). Simple sample size calculation for cluster-randomized trials. International Journal of Epidemiology, 28(2): 319-326.

Hayes RJ, Moulton LH (2017). Cluster Randomised Trials. CRC Press, New York.

Hemming K, Eldridge S, Forbes G, Weijer C, Taljaard M (2017). How to design efficient cluster randomised trials. BMJ, 358: j3064.

Henao-Restrepo AM, Camacho A, Longini IM, Watson CH, Edmunds WJ, Egger M, et al. (2017). Efficacy and effectiveness of an rVSV-vectored vaccine in preventing Ebola virus disease: Final results from the Guinea ring vaccination, open-label, cluster-randomised trial (Ebola ça suffit!). 
The Lancet, 389(10068): 505-518.

Hervé C, Laupèze B, Del Giudice G, Didierlaurent AM, Da Silva FT (2019). The how's and what's of vaccine reactogenicity. npj Vaccines, 4(1): 1-11.

Heyse J, Chan I (2016). Review of statistical innovations in trials supporting vaccine clinical development. Statistics in Biopharmaceutical Research, 8(1): 128-142.

Heyse JF, Kuter BJ, Dallas MJ, Heaton P (2008). Evaluating the safety of a rotavirus vaccine: The REST of the story. Clinical Trials, 5(2): 131-139.

Hudgens MG, Gilbert PB, Self SG (2004). Endpoints in vaccine trials. Statistical Methods in Medical Research, 13(2): 89-114.

ICH (2005). Q2 (R1) validation of analytical procedures: Text and methodology. International Conference on Harmonization, Geneva (https://www.fda.gov/media/71725/download).

Jennison C, Turnbull BW (1999). Group Sequential Methods with Applications to Clinical Trials. Chapman \& Hall/CRC, New York.

Jiang S (2020). Don't rush to deploy COVID-19 vaccines and drugs without sufficient safety guarantees. Nature, 579: 321.

Kahn R, Rid A, Smith PG, Eyal N, Lipsitch M (2018). Choices in vaccine trial design in epidemics of emerging infections. PLoS Medicine, 15(8): 1-12.

Kanaan MN, Farrington CP (2002). Estimation of waning vaccine efficacy. Journal of the American Statistical Association, 97(458): 389-397.

Kindt TJ, Goldsby RA, Osborne BA, Kuby J (2007). Kuby Immunology. Macmillan, New York.

Klein NP, Bartlett J, Rowhani-Rahbar A, Fireman B, Baxter R (2012). Waning protection after fifth dose of acellular pertussis vaccine in children. New England Journal of Medicine, 367(11): 1012-1019.

Knight-Jones T, Edmond K, Gubbins S, Paton D (2014). Veterinary and human vaccine evaluation methods. Proceedings of the Royal Society B: Biological Sciences, 281(1784): 20132839.

Kulldorff M, Davis RL, Kolczak M, Lewis E, Lieu T, Platt R (2011). A maximized sequential probability ratio test for drug and vaccine safety surveillance. Sequential Analysis, 30(1): $58-78$.

Lauer SA, Grantz KH, Bi Q, Jones FK, Zheng Q, Meredith HR, et al. (2020). The incubation period of coronavirus disease 2019 (COVID-19) from publicly reported confirmed cases: Estimation and application. Annals of Internal Medicine, 172(9): 577-582.

Le TT, Andreadakis Z, Kumar A, Roman RG, Tollefsen S, Saville M, et al. (2020). The COVID19 vaccine development landscape. Nature Reviews Drug Discovery, 19: 305-306.

Leroux-Roels G, Bonanni P, Tantawichien T, Zepp F (2011). Vaccine development. Perspectives in Vaccinology, 1(1): 115-150.

Levenson M, He W, Chen J, Fang Y, Faries D, Ho M, et al. (2020). The current landscape in biostatistics of real-world data and evidence: Label expansion. (To be submitted).

Lin M, Lee S, Zhen B, Scott J, Horne A, Solomon G, et al. (2016). CBER's experience with adaptive design clinical trials. Therapeutic Innovation $\&$ Regulatory Science, 50(2): 195-203.

Lipsitch M (2019). Challenges of vaccine effectiveness and waning studies. Clinical Infectious Disease, 68(10): 1631-1633.

Liu MA (2019). A comparison of plasmid DNA and mRNA as vaccine technologies. Vaccines, $7(2): 37$.

Lurie N, Saville M, Hatchett R, Halton J (2020). Developing COVID-19 vaccines at pandemic speed. New England Journal of Medicine, 1969-1973.

Mahanty S, Prigent A, Garraud O (2015). Immunogenicity of infectious pathogens and vaccine 
antigens. BMC Immunology, 16(1): 31.

Marciani DJ (2003). Vaccine adjuvants: Role and mechanisms of action in vaccine immunogenicity. Drug Discovery Today, 8(20): 934-943.

McIntosh K (2020). Coronavirus disease 2019 (COVID-19): Epidemiology, virology, clinical features, diagnosis, and prevention. Updated May 22, 2020 (https://www.uptodate.com/ contents/coronavirus-disease-2019-covid-19\#H1305971586).

Mizumoto K, Kagaya K, Zarebski A, Chowell G (2020). Estimating the asymptomatic proportion of coronavirus disease 2019 (COVID-19) cases on board the Diamond Princess cruise ship, Yokohama, Japan, 2020. Eurosurveillance, 25(10): 1-5.

Nason M (2016). Statistics and logistics: Design of Ebola vaccine trials in west Africa. Clinical Trials, 13(1): 87-91.

Nauta J (2020). Statistics in Clinical and Observational Vaccine Studies. Springer, Switzerland, 2nd ed. edition.

Negro F (2020). Is antibody-dependent enhancement playing a role in COVID-19 pathogenesis? Swiss Medical Weekly, 150(1516): 1-4.

NIAID (2020). NIH clinical trial of a vaccine for COVID-19 now enrolling older adults. National Institute of Allergy and Infectious Diseases, https://www.niaid.nih.gov/news-events/ nih-clinical-trial-vaccine-covid-19-now-enrolling-older-adults.

Nishiura H, Kobayashi T, Miyama T, Suzuki A, Jung S, Hayashi K, et al. (2020). Estimation of the asymptomatic ratio of novel coronavirus infections (COVID-19). Journal of Infectious Diseases, 25: 89-114.

NMPA (2020). Guidance on use of real-world evidence to support drug development and regulatory decisions. China National Medical Product Administration (http://www.nmpa.gov.cn/ WS04/CL2138/373175.html).

Ohmit SE, Thompson MG, Petrie JG, Thaker SN, Jackson ML, Belongia EA, et al. (2014). Influenza vaccine effectiveness in the 2011-2012 season: Protection against each circulating virus and the effect of prior vaccination on estimates. Clinical Infectious Diseases, 58(3): 319-327.

Oke J, Heneghan C (2020). Global COVID-19 case fatality rates. CEBM (https://www . cebm. net/global-covid-19-case-fatality-rates/).

O'Neill RT (1988). On sample sizes to estimate the protective efficacy of a vaccine. Statistics in Medicine, 7(12): 1279-1288.

Pardi N, Hogan MJ, Porter FW, Weissman D (2018). mRNA vaccines - a new era in vaccinology. Nature reviews Drug discovery, 17(4): 261.

Peeples L (2020). News feature: Avoiding pitfalls in the pursuit of a COVID-19 vaccine. Proceedings of the National Academy of Sciences, 117(15): 8218-8221.

Petherick A (2020). Developing antibody tests for SARS-CoV-2. The Lancet, 395(10230): 11011102.

Phan T (2020). Novel coronavirus: From discovery to clinical diagnostics. Infection, Genetics and Evolution, 79: 104211.

Plotkin S, Robinson JM, Cunningham G, Iqbal R, Larsen S (2017). The complexity and cost of vaccine manufacturing-an overview. Vaccine, 35(33): 4064-4071.

Plotkin SA (2010). Correlates of protection induced by vaccination. Clinical and Vaccine Immunology, 17(7): 1055-1065.

Plotkin SA (2015). Increasing complexity of vaccine development. The Journal of Infectious Diseases, 212(suppl_1): S12-S16. 
Prentice RL (1989). Surrogate endpoints in clinical trials: Definition and operational criteria. Statistics in Medicine, 8(4): 431-440.

Ray GT, Lewis N, Klein NP, Daley MF, Wang SV, Kulldorff M, et al. (2019). Intraseason waning of influenza vaccine effectiveness. Clinical Infectious Diseases, 68(10): 1623-1630.

Robert-Guroff M (2007). Replicating and non-replicating viral vectors for vaccine development. Current Opinion in Biotechnology, 18(6): 546-556.

Rosenberger WF, Lachin JM (2015). Randomization in Clinical Trials: Theory and Practice. John Wiley \& Sons, Hoboken, New Jersey.

Shih M, Lai T, Heyse J, Chen J (2010). Sequential generalized likelihood ratio tests for vaccine safety evaluation. Statistics in Medicine, 29(26): 2698-2708.

Shirley DAT, McArthur MA (2011). The utility of human challenge studies in vaccine development: Lessons learned from cholera. Vaccine: Development and Therapy, 2011(1): 3-13.

Singh K, Mehta S (2016). The clinical development process for a novel preventive vaccine: An overview. Journal of Postgraduate Medicine, 62(1): 4-11.

Strassburg MA (1982). The global eradication of smallpox. American Journal of Infection Control, 10(2): 53-59.

Tang X, Wu C, Li X, Song Y, Yao X, Wu X, et al. (2020). On the origin and continuing evolution of SARS-CoV-2. National Science Review, 0(0): 1-12.

Thomas SJ, Yoon IK (2019). A review of dengvaxia@: Development to deployment. Human Vaccines $\&$ Immunotherapeutics, 15(10): 2295-2314.

Tseng HF, Sy LS (2018). Use of real-world evidence to evaluate the effectiveness of herpes zoster vaccine. The Journal of Infectious Diseases, 218(suppl_2): S63-S67.

Vartak A, Sucheck SJ (2016). Recent advances in subunit vaccine carriers. Vaccines, 4(2): 2-18.

Weingartl H, Czub M, Czub S, Neufeld J, Marszal P, Gren J, et al. (2004). Immunization with modified vaccinia virus Ankara-based recombinant vaccine against severe acute respiratory syndrome is associated with enhanced hepatitis in ferrets. Journal of Virology, 78(22): 1267212676 .

WHO (2013). Correlates of vaccine-induced protection: methods and implications. World Health Organization (https://apps.who.int/iris/bitstream/handle/10665/84288/WHO_IVB_13. 01_eng.pdf).

WHO (2016). Human challenge trials for vaccine development: Regulatory considerations. World Health Organization: Expert Committee on Biological Standardization (https://www.who. int/biologicals/expert_committee/Human_challenge_Trials_IK_final.pdf).

WHO (2017). Guidelines on clinical evaluation of vaccines: Regulatory expectations. WHO Expert Committee on Biological Standardization. Geneva, World Health Organization (https: //apps.who.int/medicinedocs/documents/s23328en/s23328en.pdf).

WHO (2020a). Coronavirus disease (COVID-19) Pandemic. World Health Organization (https: //www. who.int/news-room/q-a-detail/q-a-coronaviruses).

WHO (2020b). Design of vaccine efficacy trials to be used during public health emergencies points of considerations and key principles. World Health Organisation (http://www10. who. int/blueprint/what/norms-standards/AP1_guidelines_Online_Consultation.pdf).

WHO (2020c). Draft landscape of COVID-19 candidate vaccines. World Health Organization (https://www.who.int/docs/default-source/coronaviruse/ novel-coronavirus-landscape-covid-195513b88a53a54fbb914c90ed9064f395 pdf).

WHO (2020d). Modes of transmission of virus causing COVID-19: Implications for IPC precaution recommendations. World Health Organization, March 27, 2020 
(https://apps.who.int/iris/bitstream/handle/10665/331601/WHO-2019-nCoV-Sci_ Brief-Transmission_modes-2020.1-eng.pdf).

WHO (2020e). Report of the WHO-China joint mission on coronavirus disease 2019 (COVID-19). World Health Organization (https://www.who.int/docs/default-source/coronaviruse/ who-china-joint-mission-on-covid-19-final-report.pdf).

WHO (2020a). Rolling updates on coronavirus disease (COVID-19). World Health Organization (https://www.who.int/emergencies/diseases/novel-coronavirus-2019/ events-as-they-happen).

WHO (2020b). WHO R\&D blueprint novel coronavirus: COVID-19 phase IIb/III vaccine trials synopisis. World Health Organization, February 28, 2020.

Woodcock J, LaVange LM (2017). Master protocols to study multiple therapies, multiple diseases, or both. New England Journal of Medicine, 377(1): 62-70.

Yang H, Zhang J, Yu B, Zhao W (2015). Statistical Methods for Immunogenicity Assessment, volume 85. CRC Press, Gaithersburg, Maryland.

Yue LQ, Lu N, Xu Y (2014). Designing premarket observational comparative studies using existing data as controls: Challenges and opportunities. Journal of Biopharmaceutical Statistics, 24(5): 994-1010.

Zhang J, Zeng H, Gu J, Li H, Zheng L, Zou Q (2020). Progress and prospects on vaccine development against SARS-CoV-2. Vaccines, 8(2): 153. 